Revue des patrimoines

15 | 2011

Le patrimoine des guides : lectures de l'espace urbain européen

\title{
Les guides européens et leurs auteurs : clefs de lecture
}

\section{Goulven Guilcher}

\section{(2) OpenEdition}

Journals

Édition électronique

URL : http://journals.openedition.org/insitu/499

DOI : 10.4000/insitu.499

ISSN : 1630-7305

Éditeur

Ministère de la culture

Référence électronique

Goulven Guilcher, «Les guides européens et leurs auteurs : clefs de lecture », In Situ [En ligne],

15 | 2011, mis en ligne le 05 décembre 2011, consulté le 01 mai 2019. URL : http://

journals.openedition.org/insitu/499; DOI : 10.4000/insitu.499

Ce document a été généré automatiquement le 1 mai 2019.

\section{cc)}

In Situ Revues des patrimoines est mis à disposition selon les termes de la licence Creative Commons Attribution - Pas d'Utilisation Commerciale - Pas de Modification 4.0 International. 


\title{
Les guides européens et leurs auteurs : clefs de lecture
}

\author{
Goulven Guilcher
}

Propos recueillis par Joanne Vajda le 10 décembre

\section{Constitution d'une collection de guides}

1 J'ai tout d'abord collectionné les guides de chemin de fer. C'était en rapport avec ma recherche universitaire sur les déplacements de loisirs ferroviaires et les trains de plaisir. J'ai ensuite étendu ma collection aux guides de voyage de la France, de toute l'Europe, de la Méditerranée, de l'Orient, de la Chine, de l'océanie, et même - enme cantonnant aux principaux guides - des États-Unis. Une collection se construit au hasard des découvertes. Mais j'ai suivi un plan de recherche en utilisant les bibliographies et eny sélectionnant les ouvrages les plus intéressants de mon point de vue. Mes critères, en fin de compte, sont assez communs pour faire l'objet d'un consensus général : écarter, sauf exception notable, les monographies de villes rédigées par un auteur n'ayant pas publié d'autres ouvrages sur des villes ou des pays ; écarter, sauf comme curiosité, les innombrables monographies de villes touristiques italiennes publiées en italien, en anglais ou en français.

2 Je ne me suis pas interdit cependant de retenir des guides de grands centres touristiques à forte fréquentation étrangère, comme Rome, Florence, Venise, ou Naples. De même, en France, les guides sur Boulogne ne pouvaient être écartés en raison de sa nombreuse population britannique et des flux qu'elle engendre. On peut en dire autant de Pau. Je suis parti des guides pour voyageurs anglais se déplaçant en Grande-Bretagne et en Irlande, puis en Europe.

3 Le premier corpus a été constitué par des guides des chemins de fer britanniques à l'ouverture des lignes, vers 1830, puis par les guides français sur la France parus un peu plus tard, dans les années 1840. Dans les années 1850-60, les guides spécialisés des chemins de fer anglais, au lieu de se présenter en petites séries sur deux ou trois lignes voisines, ont changé. De vastes collections sont apparues, couvrant tout le réseau, avec un 
volume par ligne principale ou grande ligne associée, y compris, et cela devient très intéressant, leur prolongement sur le continent.

Alors que les premiers volumes se limitaientparfois à une trentaine de pages, en comprenant l'historique de la ligne, les guides qui suivent donnent une description topographique précise: profil de ville, cours d'eau, monuments, châteaux, et même curiosités. Si les Guides Morton couvrent les grandes lignes britanniques et leurs prolongements en Europe en moins de 400 pages illustrées, les Guides Cassell, plus détaillés, offrent pour chaque grande ligne un volume de 300 pages, entre 1885 et 1914.

Les Guides George Measom, dans les années 1850 et 1860, frisent la démesure, avec parfois plus de 1000 pages par volume. Mais ces guides sont fort intéressants car on ne trouve nulle part ailleurs autant de détails sur les commerces et les industries des grandes villes traversées, avec des gravures fascinantes. Il s'agit, bien sûr, d'insertions publicitaires, mais cela n'enlève rien à leur caractère documentaire, en tenant compte des effets de perspective qui valorisent les volumes des ateliers ou des magasins.

\section{Ce qui fait la « valeur » d'un guide}

6 Pour un collectionneur, ce peut être simplement la très grande rareté pour un titre précis. Mais le collectionneur et le chercheur s'accordent dès lors que le guide n'est pas considéré comme un objet, mais comme un contenu : qualité des auteurs, choix pertinent et variété des itinéraires. L'exigence des contenus culturels et historiques se double d'une demande croissante de détails pratiques utiles concernant les déplacements, le choix des hébergements et des curiosités à visiter.

7 La qualité principale pour un guide, àmon sens, est de s'intégrer à un ensemble, de faire partie d'une collection. En plus des grandes collections bien connues, j'ai découvert des séries de titres de guides de voyage au caractère moins solide, moins massif, et moins exhaustif, mais tout à fait dignes de retenir l'intérêt par leur approche originale. On reviendra sur ces collections non pas mineures, mais moins importantes.

Bien entendu, les grandes collections sont indispensables en ce qu'elles offrent un excellent maillage de l'espace européen, chacune à leur manière et à peu près à la même époque. Elles sont donc irremplaçables et l'idéal serait de disposer de toutes les éditions pour étudier leur évolution. Mais aucune bibliothèque ne possède une telle collection. Et la crise économique a causé le sabordage d'un projet concernant Murray qui s'annonçait très prometteur. Il s'inscrivait dans la logique commerciale d'un éditeur. Peut-être les nouvelles technologies offriront-elles les moyens de réaliser des entreprises de ce genre.

\section{Le contexte d'apparition des collections de guides}

Les premières collections apparaissent au début du XIX ${ }^{e}$ siècle. Elles sont issues de l'exploitation en volumes séparés - par pays, ou par groupes de pays voisins - du premier grand guide de voyage moderne, rédigé par Heinrich August Ottokar Reichard (conseiller de guerre du Duc de Saxe-Gotha), publié en 1784 en allemand, et traduit en 1793, sous le titre :Guide des voyageurs en Europe. Ses multiples éditions intégrales en trois volumes, témoignent de son succès. Reichard "guide " véritablement dans toute l'Europe qu'il a parcourue en tant que diplomate. Le premier volume regroupe une masse considérable de conseils très précieux aux voyageurs, dont bon nombre proviennent de l'Essai pour diriger 
et étendre les recherchesdes voyageursdu Comte de Berchtold, paru en 1797 à Paris, mais dont la première édition en anglais avait été publiée à Londres en 1789 .

Après les guerres napoléoniennes et la reprise des voyages vers le continent, l'éditeur français, Hyacinthe Langloispublie un Reichard en trois ou quatre volumes, puis une collection qui grandit jusqu'à comporter onze volumes, continuée par ses successeurs Perrin et Bastin.

11 Vers 1818, l'éditeur anglais Samuel Leigh lance une collection de qualité de guides de petit format à l'ancienne, comme Langlois. Elle comprendra, elle aussi, onze volumes et s'interrompt vers 1840. Ses Reichard sont traduits du français.

12 L'éditeur Galignani, qui publie à Paris en langue anglaise, donne aussi, jusque vers 1836, le même nombre de titres de guides, souvent inspirés de Reichard. Il publie également un Manuel de la Conversation, lui aussi destiné aux touristes.

Ces trois petites collections, qui se ressemblent beaucoup, annoncent les grandes collections qui vont suivre. Les introductions comportent déjà des développements sur l'histoire, l'art, l'industrie du pays concerné, ainsi que sur les mœurs et les coutumes de ses habitants. Les itinéraires, en revanche, oscillent entre la succession d'énumérations aride et les récits très chargés deréférences historiques. Surtout, alors qu'on y trouve des indications de caractère général sur l'hébergement et la restauration, aucun ouvrage ne se risque à recommander des auberges ou des hôtelleries. Cela n'est pas encore entré dans la tradition du guide de voyage. On peut en donner au moins une raison bien simple : dans ce domaine les changements sont rapides alors que les guides sont faits pour durer un certain nombre d'années. Ils ne connaissent pas la succession rapide des éditions, caractéristique des grandes collections ultérieures.

Richard occupe une position intermédiaire, en publiant vingt-deux guides, peu à peu, entre 1822 et 1836. Maison reprend son œuvre en la développant. Mais le facteur décisif est l'arrivée de Joanne, en 1850, l'éditeur étant racheté par Hachette en 1855.

Les Guides Richard-Maison sont en quelque sorte la préhistoire de Joanne. Celui-ci révise ou refait complètement les guides existants et leur donne un style moderne qui leur permet de se hisser au niveau de la production deses grands rivaux européens.

On ne peut réduire le monde des guides aux collections. Certains titres isolés, comme leGuide Ebel du voyageur en Suisse, sont si complets - quatre volumes dans son édition intégrale au début du XIX ${ }^{e}$ siècle - qu'il est incontournable pour le voyageur spécialisé. Tous les auteurs de guides y puisent allègrement en reconnaissant en général leur dette.

17 Même si la collection devient un lieu rassurant et familier pour le voyageur, il lui fera des infidélités, à l'occasion, pour lesguides spécialisés sur l'Italie des éditeurs Vallardi, Giegler ou Artaria. Les grandes collections Murray et Baedeker ont précédé le développement des chemins de fer sur le continent. Les deux auteurs-éditeurs ont senti qu'une demande suffisante existaitde la part des touristes, malgré les difficultés de transport de l'époque. Assez rapidement, le transport ferroviaire a gonflé les flux de voyageurs. Joanne, lui, a débuté sa collection en 1850 chez Maison, en 1855 chez Hachette, alors que les déplacementsen Europe étaient devenus plus aisés, grâce à ce moyen de transport. 


\section{Ce qui distingue les trois grandes collections européennes des guides du XIXe siècle : Murray, Baedeker, Joanne}

\section{L'aspect extérieur}

La première caractéristique des trois grandes collections européennes de guides est qu'elles s'efforcent de présenter un aspect extérieur similaire, alors qu'elles sont en concurrence. Elles visent toutes à la sobriété, adoptant un format moyen uniformein-18, qui devient la norme pour les principales collections de qualité, laissant les plus petits formats aux séries populaires. Ce format est en rupture avec les guides romantiques et du début du XIX ${ }^{\mathrm{e}}$ siècle, de plus petites dimensions, mais en général plus épais et reliés plein cuir, le plus souvent par leursacheteurs. Nos trois grands éditeurs écartent le cuir, qui n'était d'ailleurs pas la présentation éditeur.

Murray, suivi par les deux autres, opte pour la percaline, dès le début, en 1836, pour Northern Germany. La teinte est rosée. Les deux volumes suivants sont verts. Puis le coloris rosé est adopté définitivement. Baedeker utilise brièvement un cartonnage imprimé dit «à la cathédrale », gothique en quelque sorte, puis copie très vite Murray en percaline rouge, et de façon définitive à partir de 1857. Lecartonnage toilé de Murray est relativement fragile. Il prend facilement l'eau et se détériore assez rapidement si le voyageur ne prend pas de précautions. On voit souvent d'ailleurs les voyageurs maudire le cartonnage Murray.

Curieusement, il n'est pas bien adapté à sa fonction et fait donc l'objet de nombreuses plaintes. Certains libraires, comme Lee, à Londres, se proposent de les relier en cuir. Murray, lui-même, propose brièvement une reliure en cuir dite « club » alors que c'est en plein air que cesvolumes souffrent des intempéries et non dans l'atmosphère cosy d'un salon.

1 La percaline de Baedeker est franchement rouge, et plus résistante. Cet éditeur reconnaît avoir copié Murray, qui n'en prend pas ombrage, et de nombreux éditeurs de guides vontsuivre, le rouge devenant une sorte de couleur standard pour les guides de nombreux pays, par exemple, Garnier et Chaix en France, Cook et Bradshaw en Angleterre, mais bien d'autres. Le libraire Lee de Londres propose des reliures en noir qui permettent deles faire passer pour des bibles ou pour des livres de messe. Ainsi, le voyageur peut entrer dans les églises et les visiter en détail ; sinon, en Italie en particulier, les guides sont saisis à l'entrée afin que les hommes d'église puissent décrire les édifices de l'intérieur et toucher une gratification pour leurs services.

Pour la très grande majorité des grands Guides Joanne, c'est la percaline bleue après une brève période de la Bibliothèque des Chemins de fer, de 1853 à 1857, qui se présentait à la fois en cartonnage rouge imprimé et en percaline noire armoriée en couleurs pour l'édition de luxe. Mais la percaline bleue existait dès 1855 chez Hachette, avec le rachat des Guides Richard dont c'était la présentation d'origine.

Le premier ouvrage de Joanne, sonItinéraire de la Suisse, publié chez Quentin en 1841, était déjà en percaline bleue. Et cela restera la caractéristique des Guides Joanne et Hachette jusqu'à nos jours où ils sont devenus lesGuides Bleus. 


\section{Le contenu} introductions en pleine page et adoptent deux colonnes pour les itinéraires (fig. $n^{\circ} 1, n^{\circ} 2$ ). Mais le confort de lecture n'est pas meilleur. Les voyageurs de l'époque ne semblent pas avoir été exigeants dans ce domaine. Les éditeurs ne pouvaient se permettre de publier une édition nouvelle chaque année de chaque titre, le coût de préparation était trop élevé en regard des ventes escomptées. Et puis l'effort devait porter aussi sur l'offre de volumes couvrant de nouvelles destinations. 


\section{Figure 1}

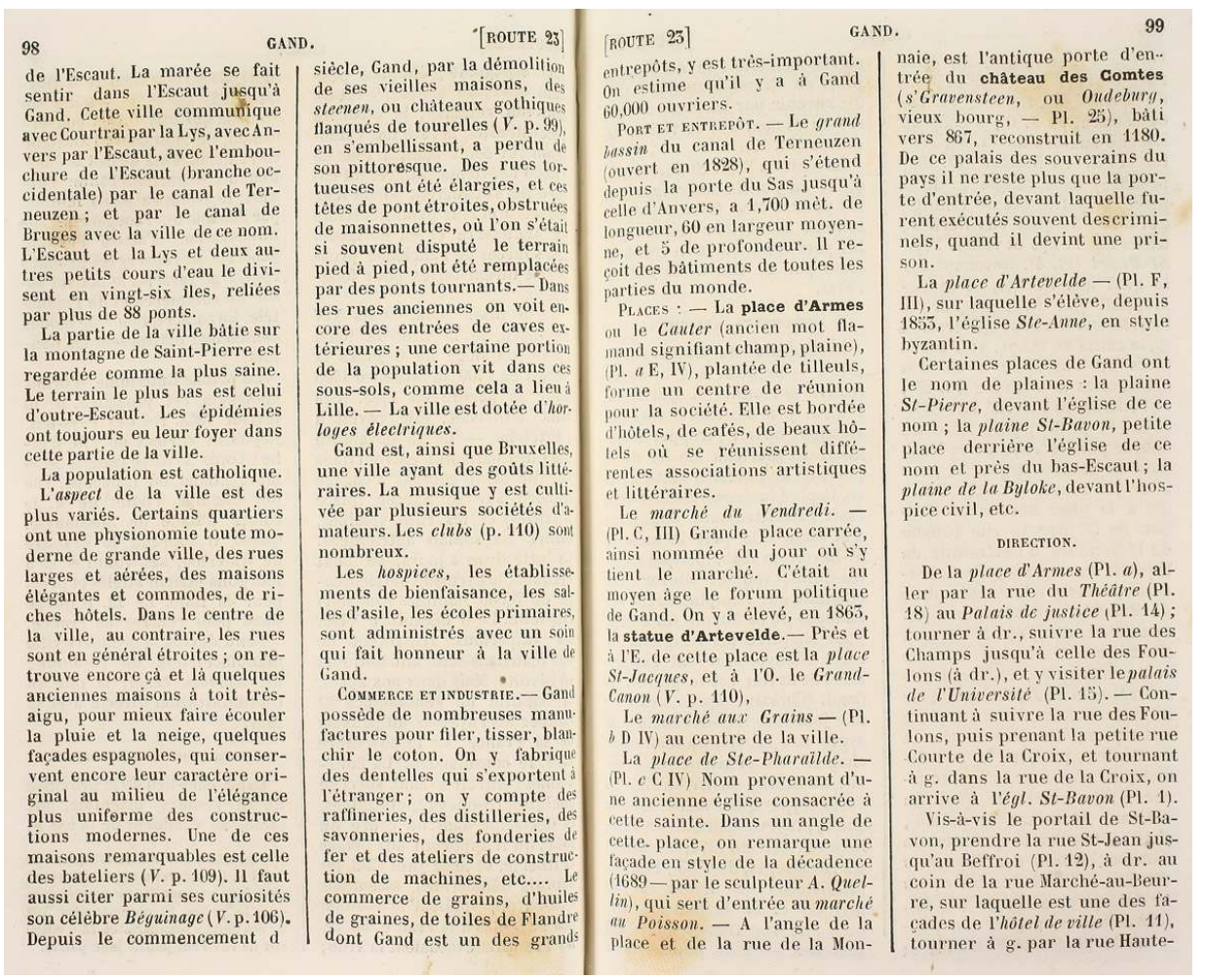

DU PAYS, A.-J. La Belgique et la Hollande. Paris : Libr. Hachette et Cie, Coll. des Guides-Joanne, Guides Diamant, 1867, p. 98-99. Coll. Guilcher. 


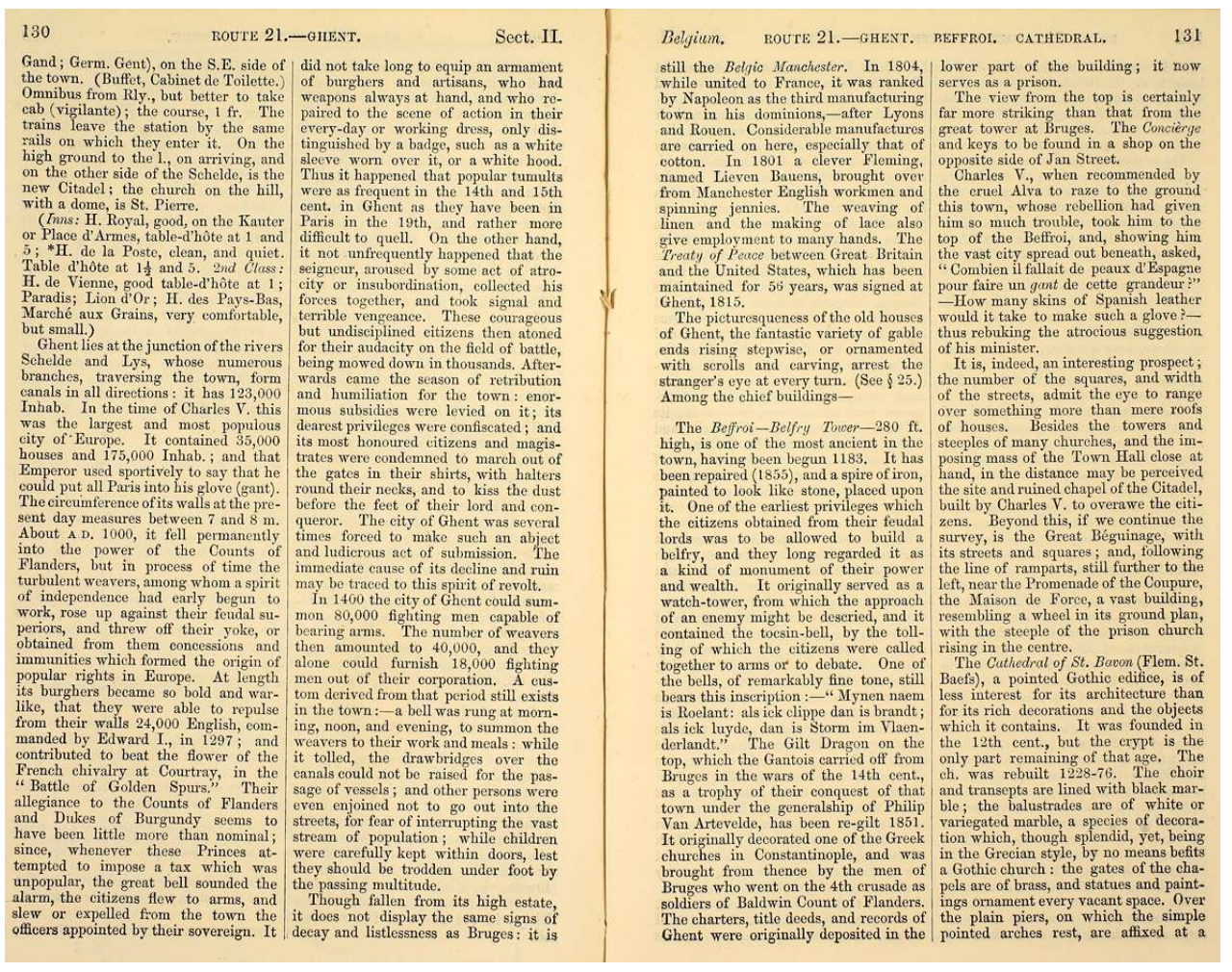

Murray's Handbook for Travellers on theContinent. Part 1. Being a guide to Holland, Belgium, Rhenish Prussia and the Rhine from Holland to Mayence, 19th ed. London : J. Murray, 1875, p. 130-131.Coll. Guilcher.

\section{Les pays traités et les langues de publication}

Murray, dans sa période hégémonique de 1840 à 1870, réédite ses guides tous les trois ou quatre ans, parfois moins souvent. Baedeker adopte un rythme plus rapide, mais lance plus tard que Murray les guides pour des destinations moins fréquentées, plus éloignées : Russie, Norvège, Méditerranée, Orient. Murray sera le seul à donner untitre sur l'Australie et le Japon. Il en produit quatre sur l'Inde contre un seul pour Baedeker. Joanne produit d'excellents guides sur l'Europe proche, mais procède avec prudence pour les autres pays.

Si l'on veut comparer les trois éditeurs, il fauttenir compte du fait que Murray s'appuie sur une clientèle aisée et nombreuse, même en ne publiant qu'en langue anglaise. Ses guides sont surtout destinés au peuple le plus voyageur. Et l'on peut se procurer le Murray chez plus de 150 agents ou dépositaires dans tous les pays d'Europe, à Malte, à Constantinople et en Grèce. Baedeker vient ensuite. Mais après 1870, il supplante Murray. Son succès tient à une conjonction de raisons : un contenu plus pratique, une offre de titres plus large et un enrichissement régulier du nombre des itinéraires. Non seulement il offrira 40 titres en allemand, mais les titres en français débutent en 1846 et il y en aura 25 différents. En langue anglaise, il y en aura 32 alors que Baedeker fait ses débuts, pour cette clientèle,seulement en 1862. Le choix d'éditer en trois langues est audacieux, mais il assied le succès de Baedeker. 

spécialisation. Finalement, sur les pays étrangers, on ne trouve guère plus d'une quinzaine de titres à la fois, Joanne se limitant aux pays les plus fréquentés par les Français. Ces titres sont certes réédités, mais l'offre n'est pas comparable avec cellesde Murray et de Baedeker. En fonction de sa destination, le voyageur n'a parfois le choix qu'entre les deux concurrents de Joanne et seulement Baedeker s'il ne pratique que la langue française. alphabétique des lieux, Joanne procède autrement. Ses index incluent et regroupent les renseignements pratiques détaillés pour chaque ville, hôtels, théâtres, moyens de transport, voire journaux, consulats etc. Ceci permet une remise à jour rapide et économique du guide sans refonte du corps du texte.

Un autre auteur plus modeste, dont on reparlera, Conty, adopte le système de Joanne en le développant, avec son "agenda du voyageur », placé en fin de volume et imprimé sur papier de couleurdifférente, qui contient de multiples informations, y compris sur les commerces. La taille des notices est fonction de l'importance des localités, classées par ordre alphabétique, mais le grand détail sur les magasins dans certaines villes est nettement lesigne d'une prospection publicitaire de l'auteur sur place. À l'époque, Conty était effectivement qualifié de publiciste, les auteurs et collaborateurs des grands guides recevant des appellations plus nobles.

\section{Les autres types de guides face aux grandes collections}

Les catalogues de libraires de l'époque montrent bien que l'offre de guides de voyage au $\mathrm{XIX}^{\mathrm{e}}$ siècle ne se limitait pas aux trois grandes collections. Il faut compter avec les collections plus modestes qui les ont précédées ou ont disparucomme celle de Hyacinthe Langlois et de ses successeurs, Galignani à Paris et celle de Samuel Leigh à Londres. Dans ce cas, le développement de grandes collections a signifié l'extinction d'un modèle qui, à la fin des années 1830, n'avait déjà plus guère la faveur du public. Il restait à Galignani, par exemple, la solution de repli de se concentrer sur un seul titre,Paris, en anglais, comme toutes ses publications, en y mettant tous ses efforts, avec des rééditions extrêmement fréquentes, constamment misesà jour, et une abondance de belles gravures sur acier. De ce fait, les autres guides, y compris ceux des meilleures collections, y renvoient. 

de succès. C'est le casde celle de Napoléon Chaix, qui s'intituleLa Bibliothèque du voyageur. C'est aussi le cas de Charles Garnier qui adopte exactement le même titre pour sa collection. Louis Hachette, puis des guides de voyage sur les pays d'Europe les plus proches. 35 titres en tout, entre 1848 et 1864, comprenant un guide des États-Unis, tout à fait unique dans le domaine des guides de l'époque. D'ailleurs ses ventes ont dû êtretout à fait symboliques.

La vente desguides ferroviairesest sévèrement contrariée par le refus catégorique opposé par Hachette à leur introduction dans les bibliothèques de gare dont il s'est assuré le monopole. Or, ces ouvrages disposaient d'atouts solides : des cartonnages au moins aussi beaux que ceux de Hachette, des gravures d'excellente qualité. Mais les textes des guides sur les pays d'Europe, qui sont un peu postérieurs, s'adressent à des voyageurs pleins de fantaisie alors que l'on attend désormais des itinéraires précis avec des indications d'hôtels.Les développements historiques et artistiques généraux disparaissent et les envolées lyriques appartiennent à un autre âge.

Napoléon Chaix a pu financer ses guides avec les profits très lucratifs de ses principales activités: les horaires, les tarifs ferroviaires, ainsi que l'impression des formulaires administratifs. À sa mort, ses fils abandonnent un secteur qui n'avait sans doute pas répondu aux espérances. Les efforts n'avaient pourtant pas été épargnés, le titre sur Paris étant publié dans les trois principales langues européennes : en français, en anglais et en allemand.

La collection Garnier, riche d'une quinzaine de titres, en français uniquement, se présente en beaux volumes de percaline rouge, parfois bleue, ornés de gravures et de cartes. Apparustardivement, en 1862, ils ne durent pas au-delà de 1867, en dépit du gros effort que représente cette réalisation pour l'éditeur. Il est vrai qu'il s'agit là d'une petite partie de son activité et qu'il se rabattra ensuite sur de petits guides beaucoup plus sommaires s'adressant à un public modeste et très pressé. Les grands Guides Garnier semblent avoir manqué d'un directeur de collection qui donne des instructions générales afin que la collection adopte une certaine uniformité. Au lieu de cela, chaque auteur expose sa conception du guide dans l'introduction et l'ensemble est assez disparate, ce qui a pu décourager les lecteurs potentiels. On en dira deux mots à propos des auteurs de guides.

Francis Coghlan constitue un cas tout à fait à part. Il publie àLondres, entre 1830 et 1860, une trentaine de guides, en général chez l'éditeur Hughes, mais chez d'autres aussi, comme Baily. C'est un grand voyageur qui rédige lui-même tous ses ouvrages. Familier des bateaux à vapeur, des calèches, et de tous les autrestransports terrestres bien avant le chemin de fer, il ouvre la route pour les voyageurs aisés vers Moscou, en 1836, alors que les grandes collections ne sont pas encore apparues, surtout sur cette destination. Ses premiers guides sont modestes, depuis Londres pour la côte anglaise ou les ports du continent. Puis il s'aventure sur le domaine de Leigh, l'éditeur, avec des titres plus réduits sur l'Europe, sauf un remarquableNetherlands up the Rhine and Switzerland, en 1837. Ensuite, dans les années 1850 à 1860, il se place dans une position intermédiaire par rapport aux grands guides, ce qui lui permet de toucher une clientèle moins fortunée. Il y fait allusion dans l'introduction de sesCentral EuropeouEuropean Touristqui offrent, selon lui, en un seul volume, l'équivalent de huit titres de Murray dont ils adoptent, d'ailleurs, la percaline rosée. 
41 Les Guides Coghlan sur l'Italie sont d'excellente qualité, tant par la richesse des itinéraires que par celle des développements historiques ou artistiques, sans pour autant égaler les plus grands. En revanche, le choix des hôtels et les commentaires qui sont faits sur les établissements offrent un détail incomparable. Le ton est très personnel et explique que ces guides aient pu trouver un public intermédiaire.

42 Le fait d'être publié ou non dans la grande vague des trois principales collections européennes ne signifie pas pour autant que l'on va à l'échec. Coghlan, lui, a commencé avant les grands guides, et il a continué pendant la période. D'autres ont été publiés uniquement pendant la période des grands guides.

43 Tout est une question de positionnement social ou encore de spécialisation. Les Guides Bradshaw, publiés à Londres et Manchester, en sont une parfaite illustration. Bien que présentant au départ des similitudes avec Chaix, en ce qu'ils sont, chacun dans leur pays, des grands éditeurs d'horaires des chemins de fer et, dans une moindre mesure, au début, de guides de chemins de fer pour Bradshaw, l'éditeur anglais réussit alors que Chaix échoue. Mi-horaires mi-guide, le Guide mensuel Bradshaw de l'Europe ouBradshaw's Continental Guide, qui débute en juin 1847, est bien supérieur à son équivalent de chez Chaix avec ses itinéraires et ses nombreuses cartes.

44 Autre publication, mais annuelle, et à partir de 1857,l'Overland Guideest le compagnon indispensable de celui qui veut faire le tour du monde. Destinés à une clientèle moins fortunée, toute une série de guides sur chacun des pays d'Europe sont publiés par Bradshaw jusque vers 1899 de façon régulière, signequ'ils ont trouvé une clientèle. Moins chers que les Murray, ils offrent une belle percaline rouge en plus petit format et comportent à la fois des cartes et des gravures (fig. $n^{\circ} 3$ ). De nombreuses librairies en font le dépôt sur le continent et les guidesplus ambitieux de Bradshaw se trouvent en vente dans les grandes villes du monde entier. 


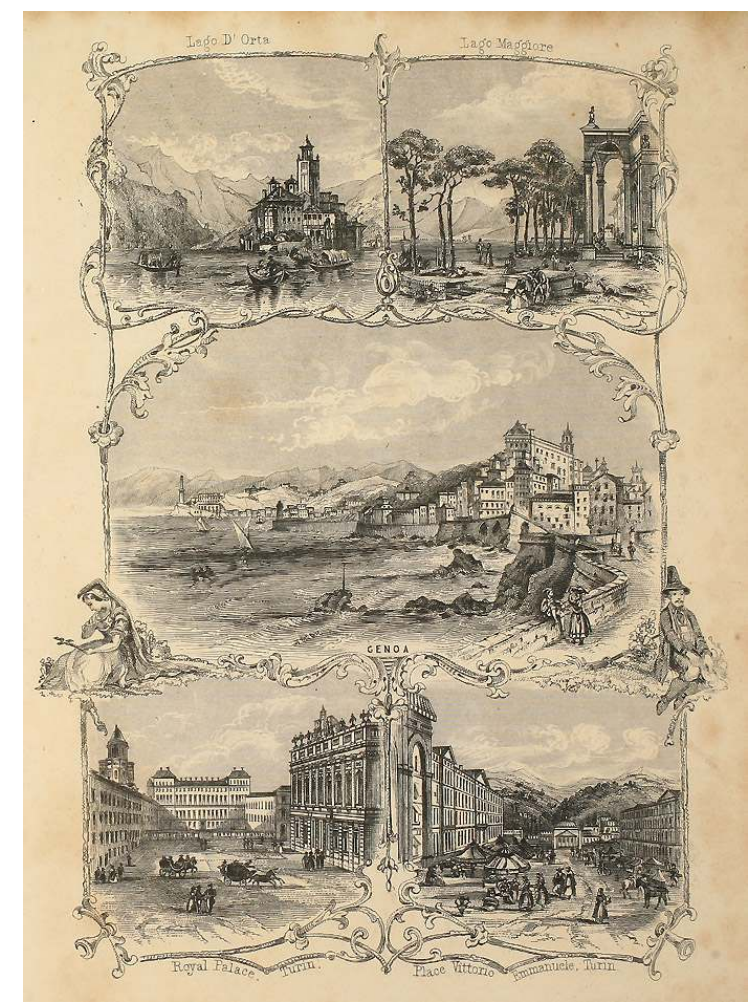

Bradshaw's illustrated Hand-Book to Italy, North and South, including Sicily and Sardinia... adapted to the railway system. London: W.J. Adams, [1867], p. 20.Coll. Guilcher.

Les Guides Black, en cartonnage vert, publiés d'abord à Édimbourg, puis aussi à Londres, sont un autre exemple de collection à succès. Cet éditeur établit sa réputation avec son Guide d'Angleterre et du Pays de Galles. Puis il ajoute un titré́cosseet un titreIrlande, ainsi que divers comtés et régions touristiques d'Angleterre en volumes séparés. Le continent n'est pas oublié avec, dans les années 1870, un très fort volume d'O'Shea sur l'Espagne et le Portugal, souvent réédité. Divers volumes plus petits couvrent progressivement, à partir de 1880 , les régions touristiques de France et d'Italie, la Hollande, la Belgique, la Suisse, et même Constantinople, Le Caire et Jérusalem. Rien de comparable avec les Murray, bien entendu, mais à leurprix modique, ils remplissent leur rôle. Les hôtels sont indiqués, des plus luxueux jusqu'aux pensions où séjourneront sans doute les lecteurs de ces volumes, imprimés sur un papier dont la qualité décline sensiblement après 1900.

Les Guides allemands Meyer'publiés entre 1862 et 1936, d'abord à Hildburghausen, puis Leipzig ont des caractères communs avec les Guides Black. Ils sont publiés en une seule langue, à la notable exception du guide suisse initié par Berlepsch - ouvrage très prisé et s'intéressent d'abord aux régions de leur pays, mais ils décrivent aussi l'Italie, l'Autriche, la France et l'Angleterre. Ils montrent leur prétentionà une certaine qualité en couvrant l'Orient et les Balkans, en plusieurs volumes, et même le voyage autour du monde,Weltreise, en un, puis deux volumes. Le guide sur Londres de plus grand format, comprend 36 superbes gravures de style romantique (fig. $n^{\circ} 4, n^{\circ} 5$ ). 
Figure 4

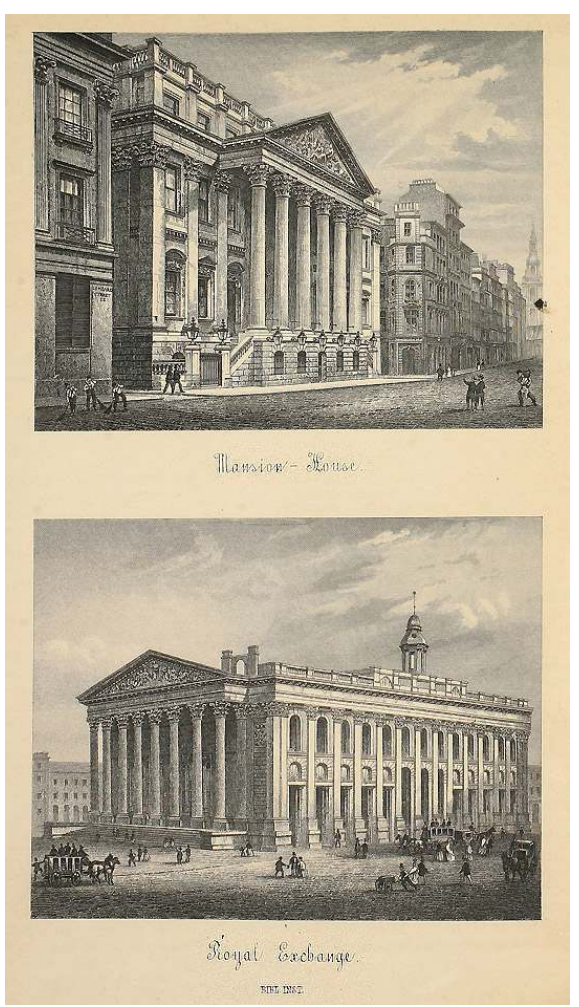

RAVENSTEIN, E.G. Meyers Reisebücher. London, England, Schottland und Irland, 3. Auflage, Leipzig: Bibliographisches Institut, 1876, p. 331.Coll. Guilcher. 


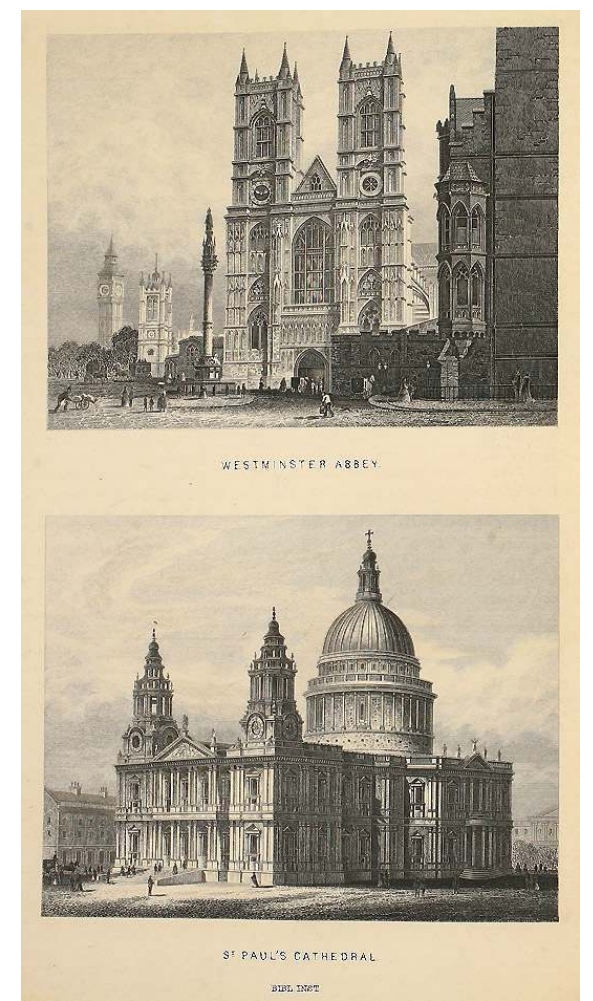

RAVENSTEIN, E.G. Meyers Reisebücher. London, England, Schottland undIrland, 3. Auflage, Leipzig: Bibliographisches Institut, 1876, p. 423.Coll. Guilcher.

Les volumes sont revêtus d'un solide cartonnage marron à titre doré, mais de format réduit. Ce sont des ouvrages d'allure très sérieuse, bien documentés, s'intéressant particulièrement aux Alpes, mais aussi aux stations balnéaires étrangères, comme à celles de la côte sud et de la côte nord de l'Allemagne. Ils sont édités par leBibliographisches Institutet comportent à ce titre une très grande richesse cartographique. Ils ne copient pas Baedeker, mais exploitent plutôt des niches comme on dirait aujourd'hui. Les hôtels sont juste cités. Baedeker finira d'ailleurs par racheter leur fonds de cartes et, à partir de 1933, le sort de Meyer est fixé. Mais c'est un hommage rendu à la qualité des Guides Meyer.

Les Anglais disposent à partir de 1874 d'une collection de qualité moyenne, sauf pour certains titres, en particulier l'Égypte. Il s'agit de celle de l'organisateur de voyages, Thomas Cook dont c'est la destination-phare etses guides pour ce pays sont des opérations publicitaires. Certains titres sont distribués gratuitement. Cook publie 25 titres, principalement sur les pays et villes d'Europe les plus visités par ses voyageurs pressés. Ce sont des volumes dans l'ensembleassez minces, en cartonnage d'abord, puis en percaline, en général rouge. Deux autres agents de voyage anglais, Gaze et Lunn, publient des guides de voyage, mais en très petit nombre et ce sont plutôt des livrets d'accompagnement. En général, les deux activités sont totalement séparées, la seule exception notable étant Cook. Ses guides sont très diffusés grâce aux multiples bureaux de l'agence Cook à travers le monde. Tous les premiers volumes sur l'Europe proche sont rédigés dans un style simple, adapté aux employés et aux membres de la petite classe moyenne qui constitue à l'époque la clientèle de l'agence. Les développements artistiques 
et historiques sont réduits à l'extrême, les itinéraires sont ceux proposés par l'agence, et la liste des hôtels est constituée par ceux qui ont conclu des accords avec Cook pour accepter ses groupes. Ils sont d'ailleurs fort nombreux.

Les descriptions de villes atteignent toujours un certain niveau. C'est la meilleure partie de ces guides qui évoluent avec l'élévation sociale de la clientèle Cook. En effet, James Mason Cook, fils du fondateur, s'adresse de plus en plus nettement à la bourgeoisie. Les guides touchent des destinations plus lointaines. Celui sur l'Égypte rivalise avec les plus grands et ceux qui abordent de nouvelles destinations en Europe, commeSpainen 1913, offrent de vastes introductions assez proches de celles des grands guides.

À son niveau intermédiaire, le Guide Cook est une réussite bénéficiant il est vrai grandement du soutien de l'agent de voyage etde la clientèle de ses voyageurs, captive en quelque sorte. Ces guides sont remaniés et simplifiés par Roy Elston à partir de 1922, ce qui assure leur survie jusque dans les années 1930.

Dans une catégorie inférieure, il faut signaler une collection allemande de petits guides qui comprend de très nombreux titres sur des pays ou des villes, très divers. Ce sont les Grieben très majoritairement publiés en allemand, et pour certains en français dès les débuts, vers 1853, et plus tardivement en anglais - 13 titres vers 1910-1913 particulièrement sur les stations de sports d'hiver, mais aussi sur Naples, la Riviera, Bruxelles et la Hollande. Ce sont des cartonnages beiges, des brochés pour les plus petits volumes. On dépasse le $160^{\mathrm{e}}$ titre en 1913. Pratiquementaucun contenu culturel dans ces guides certainement appréciés des voyageurs pour leur aspect très pratique. Les notices présentant les villes offrent un détail inconnu ailleurs sur les hôtels, des plus luxueux aux plus modestes, les hôtels garnis, les restaurants à vins, les restaurants à bière, les pâtisseries et les cafés. Pour la seule ville de Hambourg, il y en a trois pages, bien plus dans le volume sur Paris. Baedeker est beaucoup plus sélectif dans ce domaine.

Dans les Grieben, les moyens de transport et les lieux de distractions, théâtres et salles de concert, sont bien répertoriés. Les guides sont très correctement pourvus de cartes générales et de bons plans dépliants des villes principales et répondent parfaitement aux besoins des voyageurs pressés, même à Nice, Copenhague, Stockholm ou New York.

Enfin, le Français Henri de Conty est l'auteur et l'animateur de la collection qui porte son nom. Grâce à son style original qui la distingue tout à fait des grands guides, elle connaît un succès durable.Le premier volume, sur Paris, paraît en 1861, puis c'est Londres, l'année suivante, pour l'Exposition Universelle. Plus de 70 titres vont suivre. Conty disparaît en 1896, mais au XX ${ }^{e}$ siècle, Paul Mellottée poursuit la collection dans un format un peu plusgrand, avec un style plus classique.

Conty, dans ses guides au ton résolument populaire et familier, interpelle son lecteur, l'exhorte, l'encourage et le taquine à l'occasion. Il se permet même quelques petites formules un peu lestes à propos du danger des hirondelles du trottoir de Paris, ce qui est absolument exclu de tous les guides des autres collections. Coquin et badin, il accompagne le voyageur comme un ami et lui fait visiter villes et pays sans trop s'attarder sur les développements historiques. Il avoue sans détour dans sonGuide des côtes de Normandie: «J'ai pillé partout. » Mais il multiplie les conseils pratiques pour gagner du temps, économiser de l'argent en négociant au mieux partout, et prendre un maximum de plaisir en excursion. Il s'adresse aux petites bourses et ses volumes sont très attrayants avec leur percaline de couleurs variées à titres dorés. Le texte est agrémenté de gravures de monuments très classiques, mais aussi d'illustrations humoristiques qui contribuent 
beaucoup à l'aspect plaisant de cette collection fort originale. Vers 1900, cinq titres sont même publiés en anglais, choisis parmi les plus courants.

Tout à l'opposé, la collection de GuidesSilver and Company, destinée à de futurs colons ou de riches voyageurs au Canada,en Australie ou en Afrique du Sud, se taille une place grâce à son extrême spécialisation.

D'autres collections n'ont pas été retenues car elles manquaient d'envergure : trop peu de titres, ou périodes de publication trop courtes, comme pour les Guides Macmillan. Mais les principales ayant tenu leur place face aux trois grandes collections ont été répertoriées et caractérisées ici.

\section{Les auteurs de guides et leurs milieux d'influence}

Il est évident qu'une direction ferme a présidé à la genèse et au développement des trois principales collections de guides. Pour Murray, c'est John Murray, troisième du nom dans la dynastie, chez Baedeker, c'est Karl Baedeker et pour Joanne, c'est Adolphe Joanne, qui étaient, tous, de fortes personnalités. Chacun d'entre eux a payé de sa personne en effectuant sur le terrain de nombreux voyages préparatoires. Ils doivent évidemment faire des choix car le rôle de directeur de collection interdit d'être tout le temps par monts et par vaux. Par monts, c'est bien le mot pour Joanne qui est un grand montagnard, mais Murray aussi qui voyage beaucoup en Suisse.

En rédigeant les premiers guides, ils doivent déjà s'inspirer d'auteurs précédents ou faire appel à des collaborateurs pour certains aspects de certaines régions. John Murray, malgré l'intérêt qu'il porte aux guides, est requis aussi par ses fonctions à la direction d'une maison d'édition qui publie bien d'autres ouvrages : romans, récits, publications historiques. Il y aura donc plus rapidement délégation de pouvoirs chez Murray avec un auteur chargé de rédiger un titre donné.

Des archives ont été conservées à Albemarle Street à Londres et admirablement dépouillées par William Lister ${ }^{2} q u i$ a effectué des recherches sur tous les collaborateurs ayant participé aux Guides Murray. À la fin de sa bibliographie, il consacre 100 pages à un dictionnaire biographique des auteurs. C'est une véritable mine, d'autant qu'en général les archives des éditeurs ont disparu, même s'ils ont cessé leur activité à une période relativement proche.

Il faut souligner aussi que bien des guides ne portent pas de nom d'auteur, ils sont signés d'un pseudonyme. Souvent, le directeur de la collection, préfère conserver un caractère uniforme en se présentant comme éditeur, auteur de chaque titre. Il faut aussi compter avec des auteurs qui écrivent des guides pour des raisons alimentaires, maisne tiennent pas à voir leur nom mêlé à une littérature qu'ils jugent inférieure à celle qu'ils publient d'habitude. Cela déprécierait leur plume et nuirait à leur réputation.

61 Le répertoire des auteurs de Murray confirme de façon extrêmement documentée l'impression que donne la lecture des guides. Ce sont des gens aisés, cultivés, exerçant leur activité dans des domaines très variés, ingénieurs, artistes, diplomates, militaires, linguistes, botanistes, juristes, archéologues et hommes d'église. La plupart ont en commun, évidemment, de voyager beaucoup ou d'avoir occupé des postes à l'étranger. C'est à eux que l'on doit le remarquable contenu culturel des guides qui couvrent tous les domaines: historique, politique, flore, monuments, curiosités, paysages, etmême 
cynégétique. Ces auteurs décrivent ce qui les intéresse pour un public appartenant à leur milieu social.

Les défenseurs de Baedeker ont souvent tendance à exagérer le contenu trop riche des Murray qu'ils estiment pesant par rapport à leur guide modèle. Certes Baedeker est plus pratique, plus facile à utiliser et fournit une sélection très sûre d'hôtels, ce qui ne sera jamais une caractéristique de Murray. Mais il présente aussi un contenu culturel qui peut être massif.

Le Guide Baedeker est produit bien plus directement que le Murray par cette famille d'éditeurs. Karl rédige lui-même de nombreux titres d'après ses incessants voyages en Europe entre 1832 et sa mort survenue en 1859. Ensuite, ce sera Ernst jusqu'en 1861, Karl II jusqu'en 1878 et Fritz jusqu'en 1925. Ils doivent, de toute manière, faire appel, comme Murray, à une équipe d'érudits. Toutes les archives de Baedeker ont disparu dans le bombardement allié de Leipzig, le 3 décembre 1943. Mais de nombreuses contributions sont signées dans les volumes et les introductions mentionnent qu'il a été fait appel à un grand nombre de spécialistes ou d'érudits locaux. Les longues introductions sur l'art grec ou l'art italien ont été confiées à des professeurs d'université. Quant au volume sur l'Égypte, à partir de la quatrième édition, il est entièrement réalisé par le professeur Steindorff, de l'Université de Leipzig. L'éditeur le présente, non sans raisons, comme un guide pratique et sérieux pour les touristes et même pour les savants.

Joanne voyage beaucoup, mais il revendique pleinement dans les introductions des ouvrages qu'il signe ou qu'il confie à un auteur de ses connaissances le recours à une large équipe de correspondants, d'érudits locaux, ainsi que la dette contractée auprès de nombreux auteursprécédents. Le Club alpin français est largement sollicité par celui qui en a été l'un des fondateurs, puis le président et dont il reste membre actif. Les collaborateurs sont souvent des auteurs publiant régulièrement dans l'Illustration, fondée par Joanne, avec Charton, donc des gens de lettres, ou au moins des plumes de qualité reconnue.

En fin de compte, il est fait appel au même type de spécialistes que ceux qu'on a mentionnés pour Murray. Hélène Morlier ${ }^{3}$ les énumère dans sa monographie sur Joanne : historiens, archéologues, géographes, linguistes, économistes, érudits locaux, montagnards, etc.

Les auteurs des guides des trois grandes collections présentent donc les mêmes caractéristiques : gens cultivés, de milieu social aisé qui ne rechignent pas à reconnaître leur dette aux auteurs antérieurs, y compris de guides concurrents auxquels on rend hommage au passage. Ces érudits fournissent au lecteur de longues listes bibliographiques, ce qui indique bien à quel type de public ils s'adressent.

Les Guides Bradshaw sur l'Europe, qui viennent après les trois grandescollections citées au point de vue qualité, ne sont pas toujours signés. Cependant, un certain nombre des auteurs nous sont connus. Il en est parmi eux qui ont fait partie de l'équipe éditoriale de Murray. Savants ou lettrés, ils ont donné des textes assezriches, même si la présentation est relativement médiocre ainsi que l'impression. On y trouve tout de même des bibliographies, pas seulement sur le voyage, mais sur la littérature. Des exposés historiques ou artistiques recourent souvent aux chronologiesou aux listes d'œuvres, signe d'adaptation à un lectorat moins familier avec de longs exposés.

Deux collections de qualité déjà citées ont connu l'échec dans les années 1850-60 : celles de Napoléon Chaix et Charles Garnier. Les Guides Chaix n'étaient pas signés et toutes les 
archives de la maison ayant disparu vers 1960, il est très difficile d'identifier les auteurs. Ils ont le style de littérateurs : leurs textes ne présentent pas la précision ni le caractère pratique qui ont pu faire le succès des autrescollections. Quant aux Guides Garnier, de meilleure qualité que les Guides Chaix, ils sont bien signés par leurs auteurs, hommes de lettres, mais ils n'apportent pas non plus les renseignements utiles attendus par les voyageurs. "C'est comme en quelque sorte de la littérature de salon, alors que l'un des auteurs souhaitait offrir une véritable encyclopédie géographique, historique, artistique et industrielle. » C'est ainsi que s'exprime Eugène d'Auriac dans l'introduction de son volume sur la Belgique paru en 1864 (p. 8). D'autres volumes sont plus secs, celui de Simon sur l'Allemagne, l'auteur revendiquant ce style qui a pu rebuter, tandis que William Darcy sur l'Angleterre semblait plus attrayant.

\section{La pratique matérielle du voyage à travers les guides}

69 Lesguides permettent de nous représenter les conditions dans lesquelles les voyageurs effectuaient leur périple. Au fur et à mesure des éditions successives apparait la grande révolution des transports, introduite par la naissance et le développement des chemins de fer. Les yachts à voile sont supplantés par les yachts à vapeur pour les croisières en Méditerranée, de même que les transports routiers n'occupent plus la place prééminente qui était la leur dans les quarante premières années du XIX ${ }^{e}$ siècle.

70 Les utilisateurs des Guides Langlois, Samuel Leigh ou Galignani allaient de relais de poste en relais de poste et logeaient dans des auberges. Le premier Guide Murray sur la France, publié en 1843, précédait lui aussi les chemins de fer.

71 En 1827, Galignani considère qu'un tour en diligence incluant les principales curiosités dignes d'être vues en France prend trois mois et coûte cent livres sterling : on va à Paris, puis on descend vers le Sud. Encore cette estimation suppose-t-elle de voyager de façon économique, si l'on peut dire. Les mois d'août à octobre sont recommandés. Par les voies navigables, les frais sont moins importants, mais c'est nécessairement plus lent. Le tour de la Loire prend un bon mois et revient à 40 ou 50 livres, soit la moitié. À cette époque, les haltes fréquentes et le recours quasi obligatoire aux diligences imposent de prévoir une dépense d'une guinée par jour (un peu plus d'une livre). Ce sont là des frais considérables qui réservent la possibilité d'entreprendre ces voyages aux classes sociales élevées. La durée de ces déplacements implique aussi de disposer de beaucoup de temps libre. Ni Galignani, ni Murray ne cachent l'inconfort des diligences et l'état des routes.

Murray et Baedeker décrivent avec précision ce qui attend le voyageur dans les lieux d'hébergement des principaux pays d'Europe en distinguant entre les auberges situées sur les grands itinéraires, les hôtels des grandes villes et les établissements qui se trouvent à l'écart des principaux circuits. Ces derniers sont absolument déconseillés aux touristes et surtout aux dames, en raison de leur crasse et de leur mépris total de l'hygiène!

73 Les toilettes sont particulièrement critiquées dans les guides britanniques car leur état laisse vraiment à désirer, que ce soit en Bretagne, en Espagne, ou dans les auberges suisses.

74 On donne de multiples conseils sur la pratique des pourboires, les Anglais ayant la réputation d'avoir gâté des populations entières, Italiens ou Égyptiens, par leur trop grande générosité. Les négociations avec les hôteliers sont aussi une affaire délicate et les 
guides indiquent quand il faut marchander ainsi que dans quelles proportions. Parmi les précautions à prendre figure celle de demander un relevé de note régulier pendant un séjour afin d'éviter toutesurprise finale.

Le financement du voyage n'est pas oublié. Il convient de disposer d'argent pendant ces voyages parfois longs. Les guides pèsent le pour et le contre des différentes solutions. Il importe d'éviter d'avoir sur soi une trop forte somme d'argent. Le nombre de brigands diminue peu à peu, même dans le sud de l'Italie et en Espagne. Mais il ne faut pas courir de risques car de nouvelles formes de vol apparaissent dans les chemins de fer.

Les lettres de crédit traditionnellement utilisées sont grevées de fortes commissions en Italie. Il vaut mieux se faire envoyer de l'argent par la poste, par mandat.

C'est dès avant le départ que le voyageur doit bien étudier le guide pour effectuer son déplacement dans les meilleures conditions matérielles. Il saura ainsi quels accessoires sont indispensables, et même où se les procurer soit à l'avance, à Londres pour un Anglais par exemple, soit une fois arrivé à destination où on lui donne les adresses des boutiques.

78 Les produits nécessaires figurent abondamment dans la partie publicitaire du guide. Il semble d'ailleurs y avoir une certaine contradiction entre l'offre pléthorique de réclames de bagages et l'exhortation récurrente des guides à en emporter le minimum pour ne pas restreindre sa liberté de déplacement. En effet, si l'on voyage avec de nombreuses malles, il y a l'obligation de procéder à des formalités où l'attente peut être longue, on peut rencontrer des difficultés à la douane (notamment pour les produits un peu délicats, comme les cigares) et certains bagages peuvent s'égarer ou être volés. Les guides soulignent tous ces problèmes et donnent des conseils précis pour faciliter les démarches auprès des compagnies maritimes ou ferroviaires pour ceux qui s'obstineraient à s'encombrer, et ils sont nombreux! Les caricatures montrent toujours les Anglais avec une foule de bagages. Cela fait d'ailleurs la fortune des porteurs.

79 Les réclames de fabricants concurrents offrent d'ailleurs un très large choix de bagages à main, aux noms pittoresques :Carpet bag,Gladstone bag,Hold-All,Keep-All,Take-All(fig. $\mathrm{n}^{\circ} 6, \mathrm{n}^{\circ}$ 7). 


\section{Figure 6}

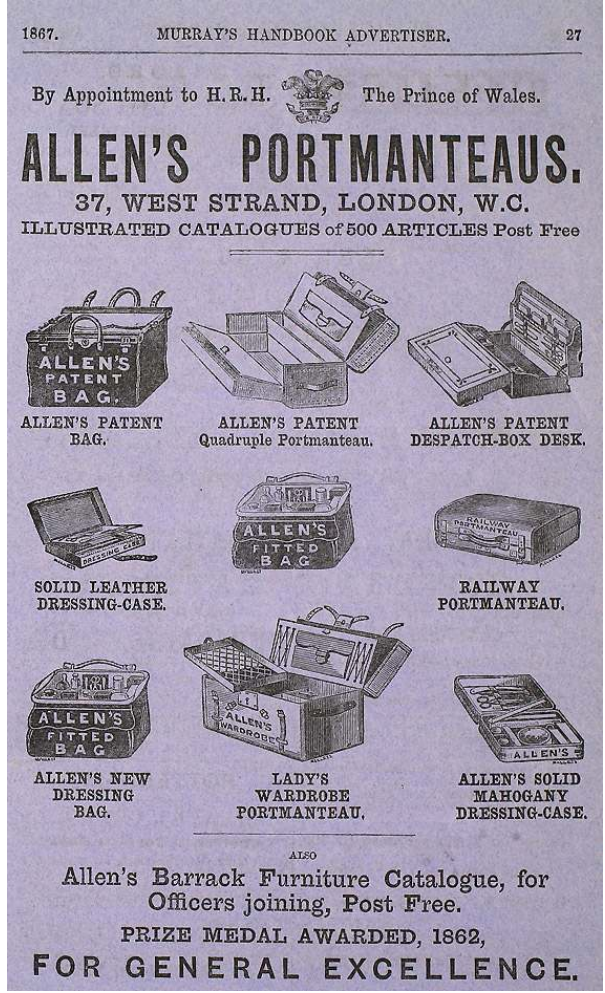

MURRAY, John. Murray's Hand Book for Travellers in France, 10th ed. London : J. Murray, 1867, p. 27 (appendice publicitaire). La même publicité figure aussi dans : Bradshaw's illustrated Hand-Book to Italy, North and South, including Sicily and Sardinia... adapted to the railway system. London: W.J. Adams, [1867], p. 41 (appendice publicitaire).Coll. Guilcher. 


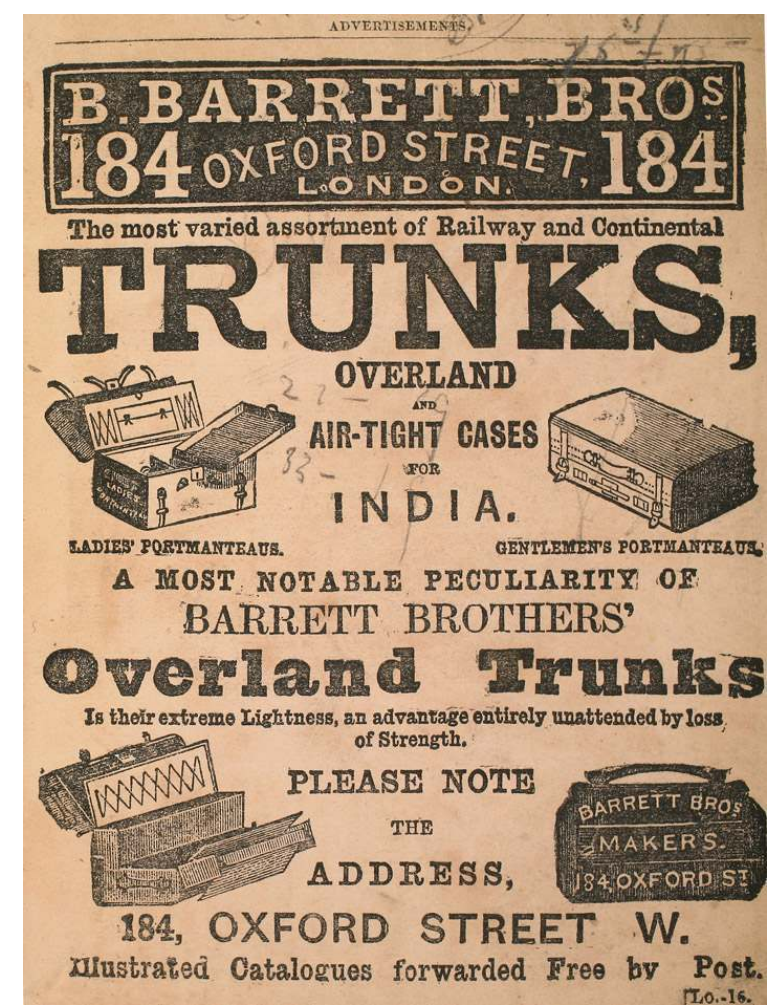

Bradshaw's illustrated Hand-Book to Italy, North and South, including Sicily and Sardinia... adapted to the railway system. London: W.J. Adams, [1867], p. 43 (appendice publicitaire).Coll. Guilcher.

En ce qui concerne les hôtels, les réclames apportent aussi un complément appréciable. Pour les bagages, on voit les différentes formes d'articles disponibles, pour les hôtels, il y a le complément visuel, des gravures représentant les façades et jardins, ainsi que l'aménagement intérieur: salons, billard, salles de restaurants et chambres. En plus des informations figurant dans le texte du guide, nécessairement succinctes, les publicités insistent sur les équipements nouveaux : éclairage électrique, ascenseur, chambre noire pour amateurs de photographie, ainsi que sur les facilités sportives à proximité, évidemment connotées socialement : équitation, golf ou tennis (fig. $n^{\circ} 8$ ). 


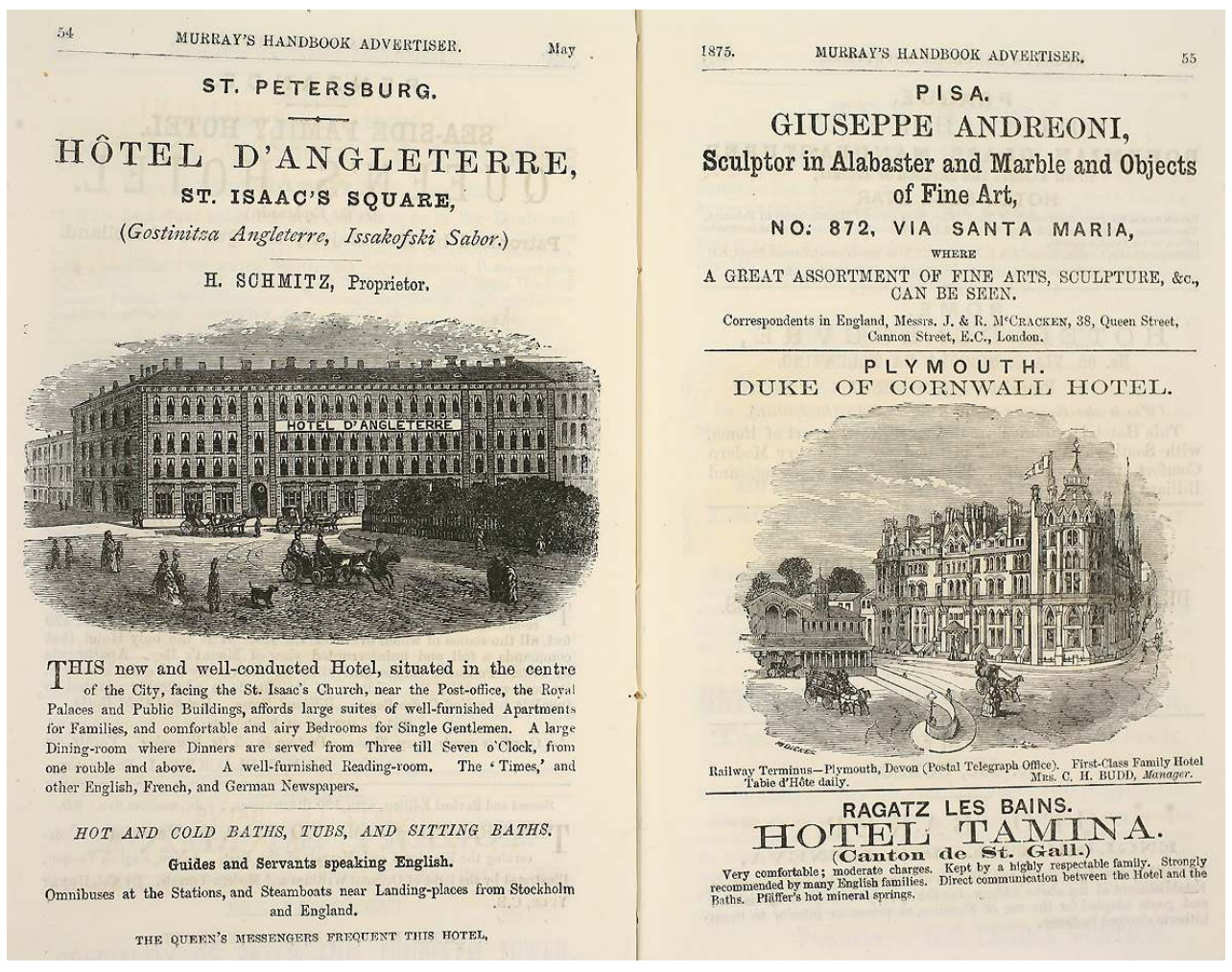

Murray's Handbook for Travellers on the Continent. Part 1. Being a guide to Holland, Belgium, Rhenish Prussia and the Rhine from Holland to Mayence, 19th ed. London : J. Murray, 1875, p. 54-55 (appendice publicitaire).Coll. Guilcher.

Selon Baedeker, un voyageur seul dépense 20 à 25 Francs par jour, 10 Francs s'il séjourne dans une ville, et ceci dans les parties les plus fréquentées de l'Europe.

Il envisage, en 1875, pour l'Italie par exemple, selon les divers types de clientèle, le court séjour à l'automne (de la mi-septembre à la mi-novembre) ou au printemps (de la mi-mars à fin mai) jusqu'aux voyages qui durent depuis le début septembre jusqu'à l'approche de l'été, l'hiver étant considéré à cette époque comme la meilleure saison pourfaire le voyage en Italie. Cela engage donc des frais considérables et demande une grande disponibilité.

Le Guide Murray de la Méditerranée donne toutes les indications pour y faire, en 1880, une croisière de huit mois en yacht de 150 tonneaux, à partir de Londres, avec 12 membres d'équipage et 5 passagers. On y trouve le relevé de tout ce qui est nécessaire pour le navire, l'équipage et les provisions pour le périple. Le total approche les 2000 livres excluant, bien entendu, les dépenses aux escales éventuelles.

L'Overland Guidede Bradshaw pour un voyage en Inde, en 1898, s'adresse au même type de clientèle ainsi qu'en témoigne l'impressionnante liste de quatre pages, deux pour les ladies, deux pour les gentlemen, dont le trousseau indispensable comprend: 30 paires de chaussettes, 36 cols de chemise, 18 cravates, vêtements de tous les jours, bottes, chapeaux, équipement de chasse, sans compter les tenues de soirée. Il s'agit là d'exemples extrêmes pour des voyages longs, vers des destinations lointaines.

Pour les relations avec le personnel des hôtels, un guide de montagne ou un drogman, dans l'Orient, rien de tel que l'aide de Murray et surtout de Baedeker. Baedeker, dans pas mal de cas, propose même de les remplacer purement et simplement. 

milieu du XIXe siècle, n'ont pas adopté la même politique en ce qui concerne l'iconographie. Leigh et Langlois produisent des guides enrichis d'une grande carte générale du pays, tandis que le corps du volume inclut une quantité généreuse de cartes régionales et de plans de villes. Les volumes étant de petite dimension, les cartes et plans, assez grands, sont pliés de multiples fois. Ce sont de bonnes productions, bien lisibles. Leigh place même une gravure en acier en tête de volume. Galignani, de son côté, se contente d'une bonne carte générale, à multiples plis, sauf pour le plan sur Paris comme je l'ai mentionné.

91 Les Guides Reichard, antérieurs à ces guides et leur ayant, pour ainsi dire, donné naissance, comprenaient un très beau volume intitulé :Atlas portatif et itinéraires de l'Europe avec neuf cartes gigantesques extrêmement détaillées et absolument magnifiques.

92 LeGuide des Voyageurs en Europene comprenait pas d'illustrations, seulement des panoramas dépliants des curiosités et des capitales, sur lesquels les curiosités sont étrangement signalées juste par des points.

Murray donne pour chaque volume une grande carte dépliante générale, des cartes de régions et des plans de villes (fig. $n^{\circ} 9$ ). Les plans de musées sont très rares. C'est un guide essentiellement descriptif. Seul le volume sur l'Égypte comporte un certain nombre 
d'illustrations, alors que l'Espagne offre quelques plansde monuments. Cette façon de procéder reste immuable pendant la durée de l'existence de la collection. Un titre tardif sur le Japon, en 1884, comporte cependant de belles illustrations. Peut-être que le sujet s'y prêtait.

Figure 9

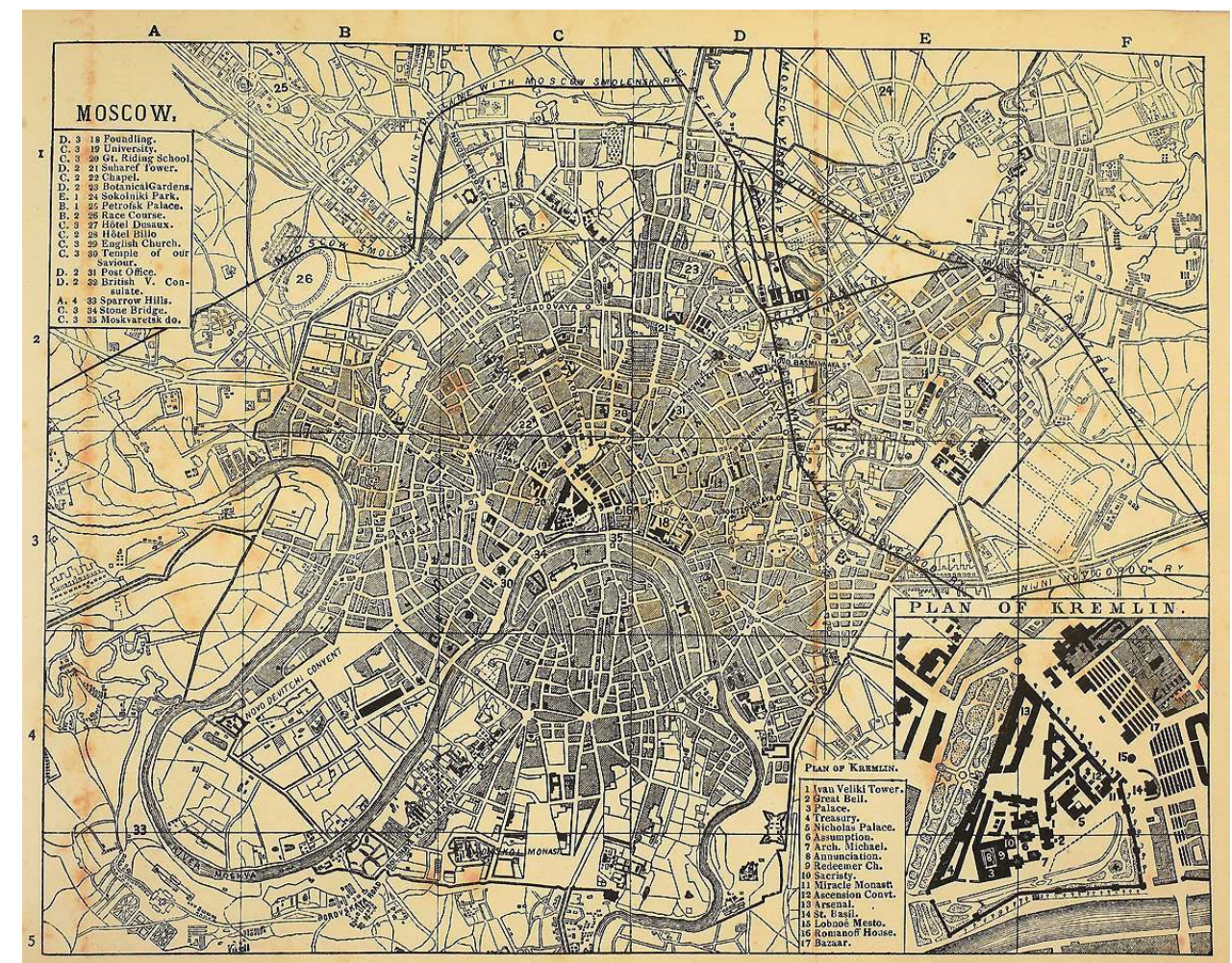

Murray's Handbook forTravellers in Russia, Poland and Finland including the Crimea, Caucasus, Siberia and Central Asia, 3rd ed. London : J. Murray, 1875, p. 222.Coll. Guilcher.

Baedeker se fait remarquer par le nombre et la qualité des cartes, des plans, et la beauté des panoramas dépliants, tandis qu'on trouve inclus dans le texte des plans d'églises et de musées (fig. $\left.{ }^{\circ} 10\right)$. Chez Baedeker, l'enrichissement est continuel. La Suisse, par exemple, comportait 10 cartes en 1863, 22 en 1874,59 en 1901 et 111 en 1928, sans compter15 panoramas. L'Égypte, titre particulièrement soigné par cet éditeur, comporte, en 1929, 106 cartes et plans et 56 gravures, le nombre de gravures étant tout à fait exceptionnel en raison du sujet, mais à cette époque le nombre de cartes a augmenté régulièrement jusqu'à dépasser la centaine pour des volumes comme l'Italie, la Norvège, l'Allemagne du Nord, l'Allemagne du Sud et la Russie. Les cartes sont très vite en couleur et, mises à jour régulièrement, elles constituent l'un des points forts des GuidesBaedeker, et l'une des supériorités sur les Guides Murray. 


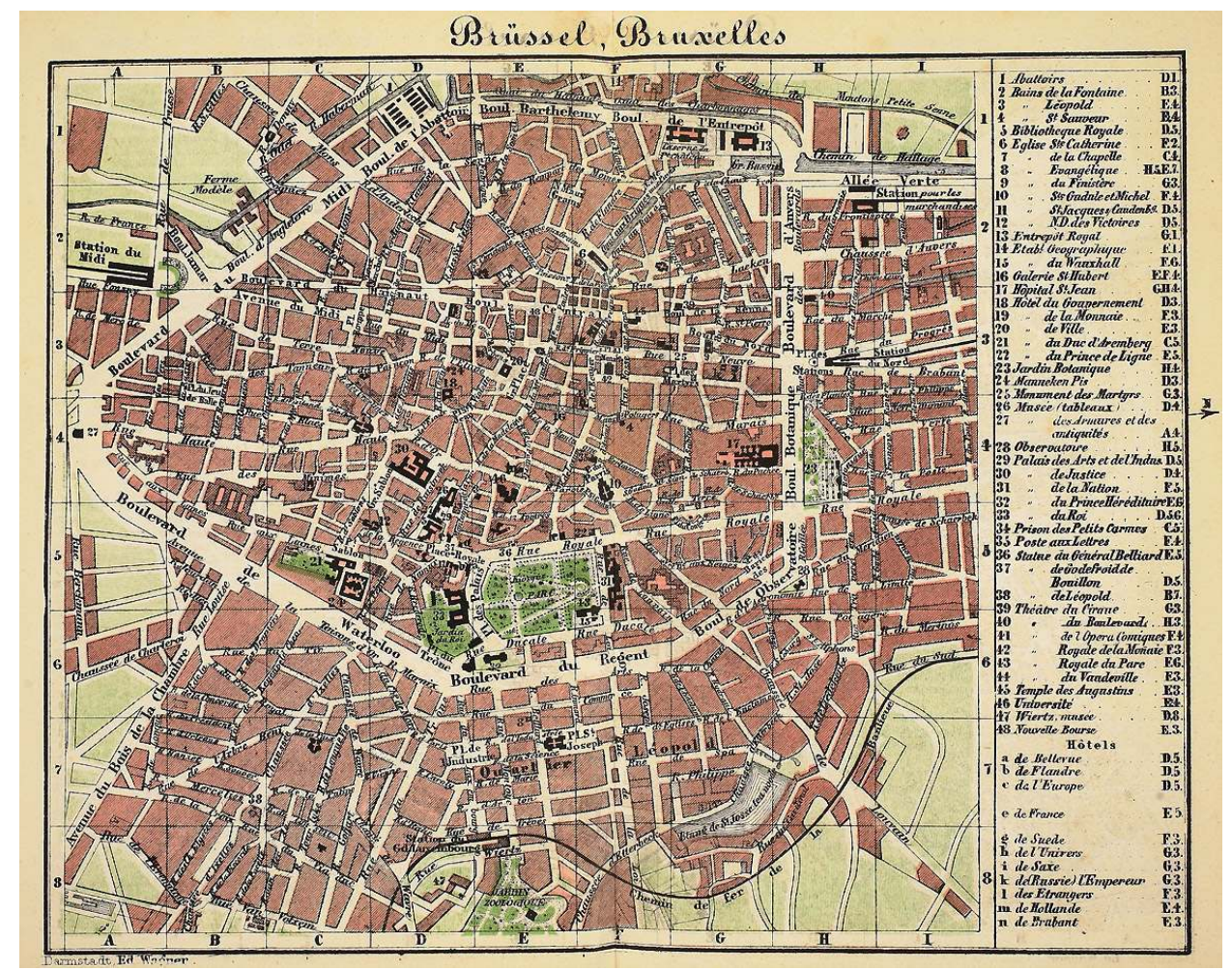

BAEDEKER, Karl. Belgien und Holland. Handbuch für Reisende, 12. Auflage, Coblenz/Leipzig : Verlag von Karl Baedeker, 1873, p. 45. Coll. Guilcher.

Dans les Guides Joanne, on ne trouve pas une telle abondance. Les volumes de la Bibliothèque des Chemins de fer comportent une carte dépliante ${ }^{4}$. On y trouve aussi denombreuses gravures sur bois. Les Guides Joanne sur les régions ou les pays, dans la suite des Guides Richard, ont en général une grande carte dépliante, souvent dans une pochette à la fin comme cela se pratiquait aussi chez Murray, Leigh, Chaix, Garnier,Conty et bien d'autres, et des plans de ville sur une ou deux pages, dans l'ensemble assez peu attrayants. Ils ne sont pas dépliants, en tout cas.

Le nombre et la qualité des plans se développent. En 1880, par exemple,la Normandie comprend beaucoup de beaux plans dépliants. L'Itinéraire de l'Orienten trois volumes est particulièrement soigné, avec cartes en couleur, plans, gravures, et même un volume supplémentaire d'atlas (fig. $n^{\circ} 11, n^{\circ} 12$ ). Pour les autres titres il n'y a pas de gravures dans le texte. 
Figure 11

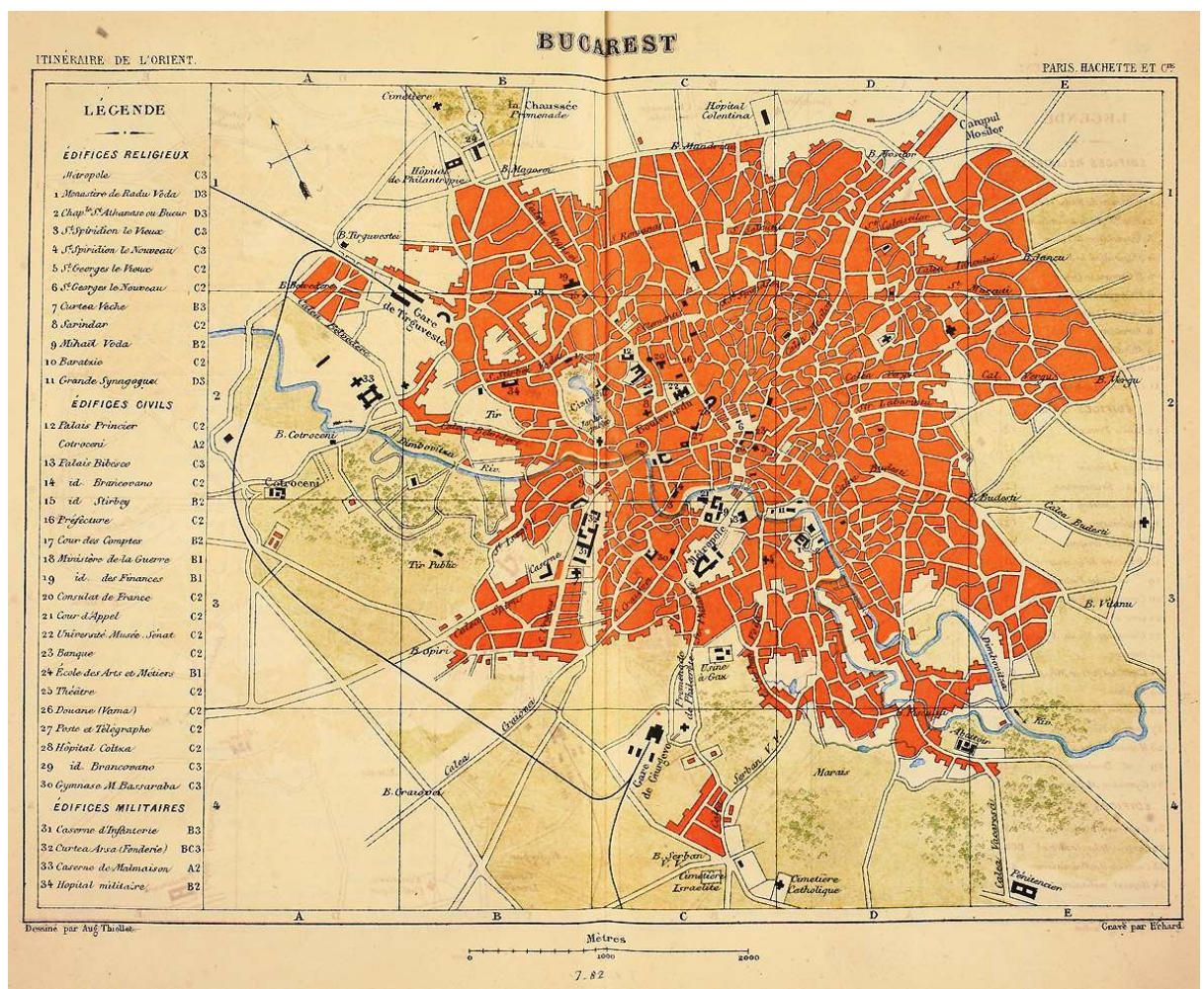

ISAMBERT, Émile. Orient, Grèce et Turquie d'Europe. Paris : Libr. Hachette et Cie, Coll. des GuidesJoanne, 1881, p. 946. Coll. Guilcher. 


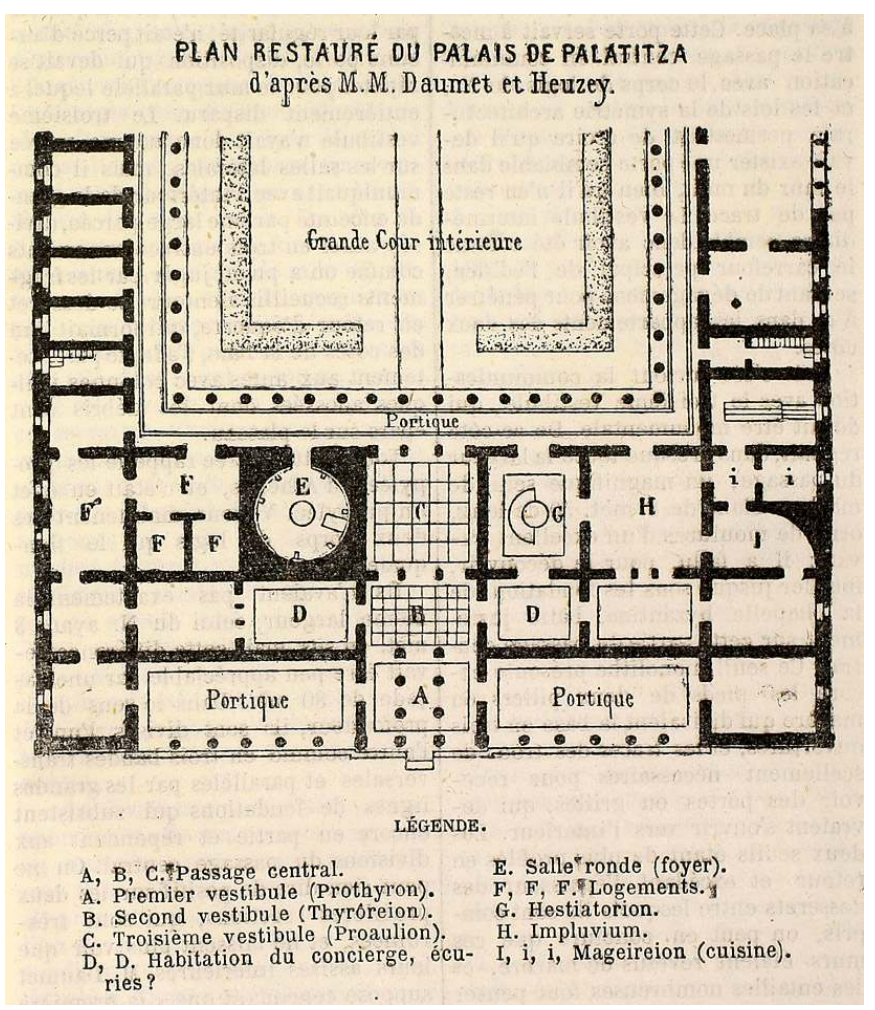

ISAMBERT, Émile. Orient, Grèce et Turquie d'Europe. Paris : Libr. Hachette et Cie, Coll. des GuidesJoanne, 1881, p. 771. Coll. Guilcher.

Les Guides Chaix et Garnier sont tous deux sur le même modèle : une carte générale et des gravures pleine page, sans évolution. C'est évidemment bien maigre comparé à Baedeker.

Le contenu iconographique et cartographique des Guides Cook est modeste au début, puis se développe au fil des années, surtout pour les destinations les plus fréquentées, comme la Suisse, l'Italie, la Riviera ou la Belgique. Il est soigné pour les pays d'Orient dont John Mason Cook fait unespécialité de la maison. Dans l'ensemble, ce qui est offert est de bonne qualité pour des guides de cette catégorie.

Bradshaw se signale dans ses guides, séparés sur les pays d'Europe, par des planches regroupant chacune plusieurs petites gravures romantiques du plus bel effet, ceci en plus des cartes et plans que l'on trouve dans les autres guides. Mais, si le texte du guide de l'Europe et celui du Tour du monde connaît un fort accroissement, il n'en est pas de même pour le nombre de cartes réparties par pays et correspondant à chaque section. Le Guide Bradshaw de l'Europe présente une autre singularité : c'est l'édition spéciale qui est enrichie en cartes, pratique inconnue chez les autres guides et qui semble un lointain écho des éditions de luxe des guides du début du XIX ${ }^{e}$ siècle, riches en gravures sur acier, alors que l'édition ordinaire ne contient qu'une seule carte, quand elle en contient une !

Dans les grands guides du XIX ${ }^{e}$ siècle, les gravures sont surtout très présentes dans les publicités, dès 1840 chez Murray, à partir de 1870 chez Joanne, alors qu'elles sont rares dans le corps du guide. À la fin du XIX ${ }^{e}$ siècle, les gravures sont souvent exécutées d'après des photographies car la reproduction de photographies exige un papier de qualité spéciale.Les guides se mettent à inclure des photographies dans le texte, Hachette en particulier, mais pas dans ses plus belles collections. 
101 Les cahiers publicitaires utilisent déjà la photographie de leur côté avec des résultats mitigés. En Angleterre, Black selance à la fin du XIX du siècle dans les illustrations photographiques hors-texte, pleine page, imprimées sur un papier bien meilleur que celui du texte, dont la qualité est devenue médiocre. Le noir et blanc sur la Suisse fait regretter l'effet de rêve que procuraient les gravures de panoramas. Les vues sur Le Caire sont assez sombres et brumeuses, quant à Jérusalem, les photographies en couleurs criantes sont un véritable désastre.

Un autre éditeur anglais qui se lance plus tardivement dans les guides sur les pays d'Europe, Ward Lock, offre vers 1912-1930 des petits ouvrages de poche bien faits avec cartes, plans et 60 photos par volume. On est vraiment entré dans l'ère du guide illustré et l'illustration va gagner sans cesse du terrain au point de réduire le texte à la portion congrue dans bien des collections modernes au caractère éphémère. Il s'agit là de la mutation majeure du $\mathrm{XX}^{\mathrm{e}}$ siècle, facilitée par les progrès de l'impression et du tirage en couleur.

\section{Les publicités dans les guides}

On a déjà évoquél'utilité des publicités, qui sont le fruit d'une collecte par l'éditeur après démarchage auprès des annonceurs. Chez Murray, la remise des annonces se faisait jusqu'au mois de mai pour chaque année. Elles étaient publiées dans un cahier annuel. L'emplacement des publicités, dans les grands guides, n'est pas sur les couvertures, car cela enlèverait de la dignité et de la noblesse. Il y a une présentation basique indispensable pour ne pas dévaluer le produit auprès de la clientèle. Le guide de voyage essaiede se dégager de son image trop utilitaire. Il doit pouvoir gagner sa place dans les rayonnages des bibliothèques, au même titre que les autres ouvrages, littéraires par exemple. Le guide doit donc passer inaperçu. Mais cela n'empêche pas la présence de publicités à l'intérieur, bien séparées du texte en général, en cahiers spéciaux, sur papier de couleur différente. On les trouve en fin de volume, parfois aussi en début de volume, lorsqu'elles concernent plutôt la région ou le pays décrit (fig. n¹3). Pourles Guides Chaix de l'Europe, c'est en début et au milieu de volume que l'on trouve les réclames. Murray les introduit très vite de façon massive, Joanne attend 1870 et Baedeker, au contraire, les juge suspectes dans un guide qui doit rester au-dessus detout soupçon et ne les introduit qu'en 1920. 


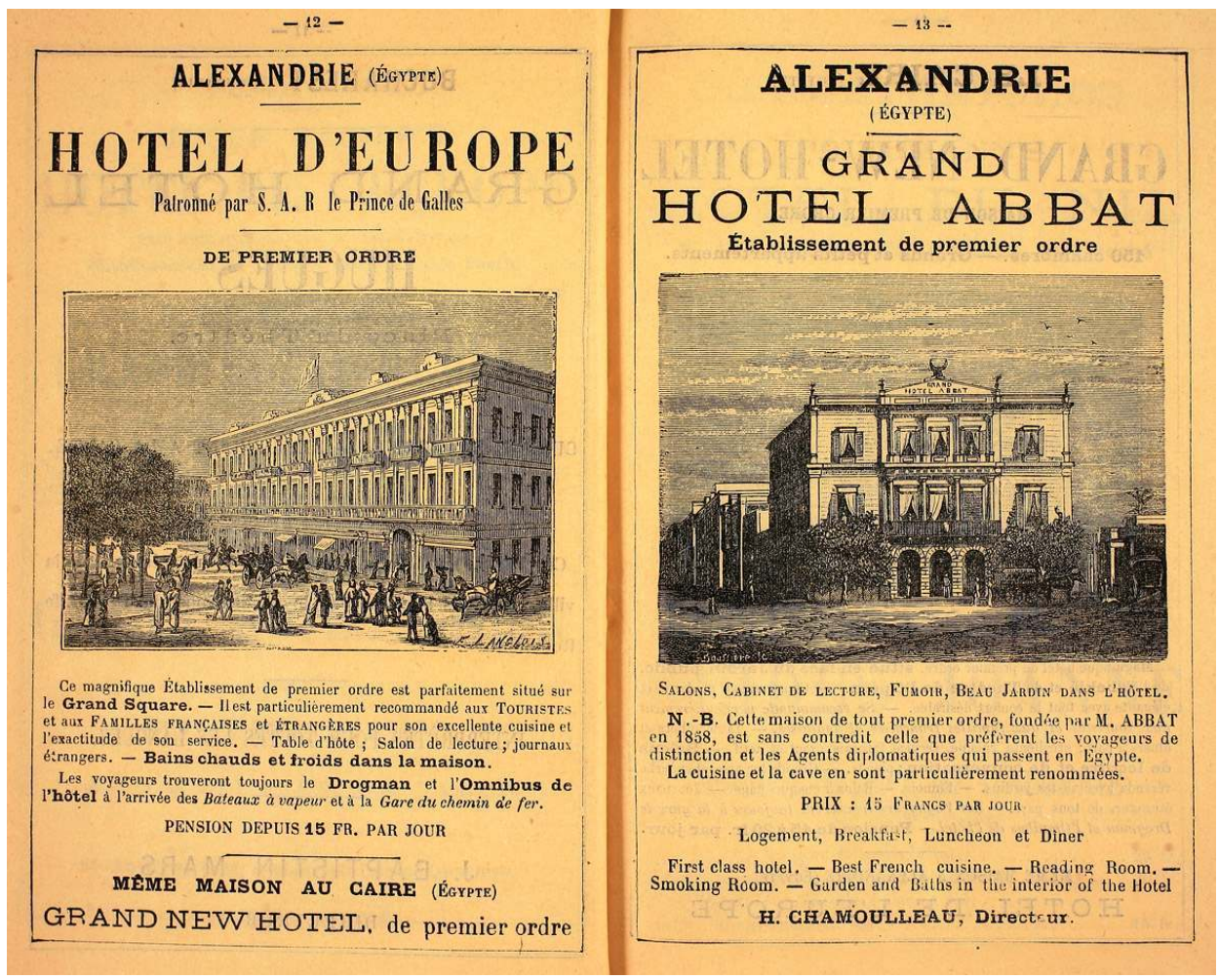

ISAMBERT, Émile. Orient, Grèce et Turquie d'Europe. Paris : Libr. Hachette et Cie, Coll. des GuidesJoanne, 1881, p. 12-13. Coll. Guilcher.

Ces scrupules ne sont pas tous fondés. En effet, les publicités sont loin d'être toutes de nature hôtelière, leur variété est considérable. Pour ces autres publicités, la contamination éditoriale n'est pas à craindre. On remarquera que pour les guides d'une même année dans une collection, on trouve un cahier commun de publicités regroupant tous les hôtels d'Europe et de la Méditerranée, classées par ordre alphabétique de villes qui ont souscrit des annonces.

5 La présentation des annonces est très variée selon les textes et les documents fournis par les annonceurs. La typographie, la mise en page, la présence éventuelle de gravures sont laissées à leur choix. Murray demandait 4 livres sterling pour une page entière et annonçait un tirage de 15000 exemplaires par an. Bon nombre de publicités couvraient une page et, par exemple, à Vienne, un groupe d'hôtels souscrivait une annonce pour une page commune.

Les publicités couvrent tous les besoins des voyageurs, des plus pratiques aux plus futiles, en dehors de l'hébergement et de la restauration proprement dits : hôtels, pensions, cafés, restaurants, ainsi que les banques. Les transports ferroviaires et maritimes sont en bonne place. On y découvre tout l'univers du voyage, avec ses accessoires (jumelles, longues-vues, etc.), bagages et ingrédients divers (eaux de Cologne, onguents, potions) mais aussi magasins de mode, bottiers, bijoutiers et marchands de colifichets (fig. $n^{\circ} 14$ ). L'éditeur du guide inclut les publicités pour ses propres ouvrages, guides ou autres. Les publicités accueillent aussi les librairies qui, en donnant un panorama de leur stock en guides touristiques, nous renseignent sur l'offre existante. C'est l'occasion de constater la 
très grande variété sur le marché, même si certains titres ne sont pas toujours facilement identifiables car ils sont brièvement mentionnés dans une longue liste.

Figure 14

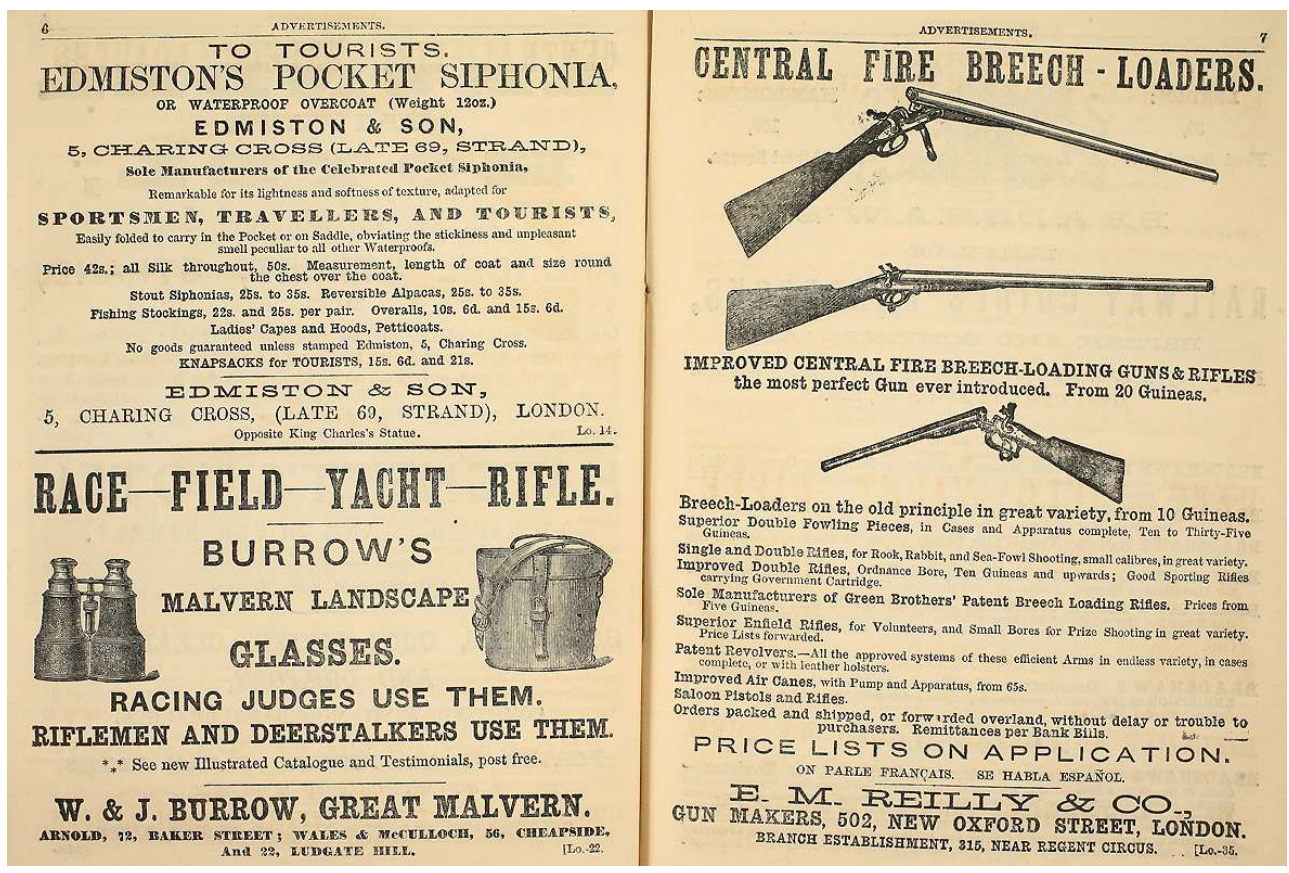

Bradshaw's illustrated Hand-Book to Italy, North and South, including Sicily and Sardinia... adapted to the railway system. London: W.J. Adams, [1867], p. 6-7 (appendice publicitaire).Coll. Guilcher. guides, à commencer par Bradshaw, qui a pourtant une excellente réputation, ou Cook, se soucient moins de l'apparence et affichent des réclames sur les pages intérieures de couverture, avec une place de choix pour la maison de deuil Jay, chez Bradshaw, ce qui constitue une curiosité pour un guide de voyage. Les guides plus ordinaires osent la publicité sur la quatrième page de couverture, ce qui les fait classer dans la catégorie des livres à bon marché. C'est le cas de la série desDickens's Dictionaries, sur Londres, Paris oula Tamise, ou des Guides Conty. Conty, publiciste, se risque aussi à la publicité 
rédactionnelle et insère des réclames dans le texte en les faisant toutes dessiner par l'artiste Uzès, ce qui donne une allure très plaisante, mais on ne sait plus très bienoù est la frontière entre le guide et la publicité.

111 On trouve des réclames à l'intérieur de certains guides, dans le texte, et même au dos des cartes dépliantes, tout espace étant monnayable. On comprendra qu'il ne s'agit pas des ouvrages appartenant aux collections les plus raffinées. Bradshaw, sur le déclin au premier quart du $\mathrm{XX}^{\mathrm{e}}$ siècle, $\mathrm{y}$ a recours.

Ainsi, le domaine des guides de voyages est-il extraordinairement riche et multiforme, car, à côté des grandes collections à diffusion internationale, qui présentent de grandes similitudes, dans la mesure où elles visent des publics assez voisins, on trouve de nombreuses autres collections plus modestes, plus typées aussi, chacune dans leur genre, qui offrent au voyageur ou au simple excursionniste un service adapté.

113 Le chercheur, lui, découvre par le biais de ces guides les multiplesfacettes de l'univers de l'époque, en particulier dans son contexte matériel, artistique, architectural, urbain ou naturel. La masse de données renfermées dans les guides et régulièrement mise à jour constitue véritablement une source irremplaçable pour lesociologue ou l'historien.

\section{BIBLIOGRAPHIE}

\section{Principaux articles sur les guides de voyage}

Une vingtaine de notices bibliographiques sur des collections de guides d'éditeurs européens au XIX $^{\mathrm{e}}$ et au XX ${ }^{\mathrm{e}}$ siècle, publiées dansLa Lettre du Marché Brassensentre 1994 et 2002, particulièrement :

$\mathrm{N}^{\circ} 38$ (26 05 1994), les Guides Michelin.

$\mathrm{N}^{\circ} 39$ (26 07 1994), les Guides Garnier.

$\mathrm{N}^{\circ} 53$ (26 11 1996), le voyage à Londres au XIX siècle.

Nº 54 (26 01 1997), les Guides Richard (I) de 1823 à 1836.

N 55 (26 03 1997), les Guides Richard (II) de 1836 à 1855.

$\mathrm{N}^{\circ} 56$ (26 05 1997), les Guides Conty.

$\mathrm{N}^{\circ} 57$ (26 07 1997), les Guides Chaix.

$\mathrm{N}^{\circ} 60$ (26 01 1998), les Guides Galignani, publiés en anglais à Paris.

$\mathrm{N}^{\circ} 61$ (26 03 1998), les Guides Langlois, Perrin et Didier.

$\mathrm{N}^{\circ} 62$ (26 05 1998), les manuels de conseils aux voyageurs.

$\mathrm{N}^{\circ} 64$ (26 09 1998), lesGuidesHeinrich August Ottokar Reichard.

$\mathrm{N}^{\circ} 66$ (26 01 1999), Madame de Genlis et sonManuel du voyageur.

$\mathrm{N}^{\circ} 68$ (26 05 1999), les manuels de la conversation en langues étrangères.

$\mathrm{N}^{\circ} 70$ (26 09 1999), les Guides Baedeker (I), Leipzig, Cologne, Paris et Londres.

$\mathrm{N}^{\circ} 71$ (26 11 1999), les Guides Baedeker (II).

$\mathrm{N}^{\circ} 72$ (26 01 2000), les Guides John Murray, de Londres.

$\mathrm{N}^{\circ} 74$ (26 05 2000), les Guides Thomas Cook, de Londres. 
$\mathrm{N}^{\circ} 76$ (26 09 2000), les Guides Madrolle.

$\mathrm{N}^{\circ} 77$ (26 11 2000), les Guides Samuel Leigh, de Londres.

$\mathrm{N}^{\circ} 78$ (26 01 2001), les Guides Francis Coghlan, de Londres.

$\mathrm{N}^{\circ} 79$ (26 03 2001), les Guides anglais Bradshaw (Londres et Manchester).

$N^{\circ} 89$ (26 11 2002), les Guides du Musée de la Poste à Paris.

\section{Autres articles}

Les guides de voyage, pistes de recherche et bibliographie sommaire, InRevue française de civilisation britannique, vol. VIII, $\mathrm{n}^{\circ} 4$, (juin 1996), p. 123-147. Recense en 235 notices les ouvrages en langue anglaise, principalement au XIX ${ }^{\mathrm{e}}$ siècle.

Les guides des chemins de fer, pratiques anglaises et françaises, In MOUREAU, F. et POLINO, M.-N. (éd). Écritures du chemin de fer,Actes du colloque en Sorbonne Association pour l'Histoire des chemins de fer en France (AHICF) et Groupe de Recherches sur laLittérature des Voyages (GRLV). Paris : Klincksieck, 1997, p. 22-36, avec bibliographie.

Naissance et développement du guide de voyage imprimé : du guide unique à la série, une stratégie de conquête des lecteurs?, In CHABAUD, Gilles, COHEN, Évelyne et autres (éds).Les Guides imprimés du XVI ${ }^{\mathrm{e}} \mathrm{au} \mathrm{XX}^{\mathrm{e}}$ siècle, villes, paysages, voyages, Actes du colloque des 3-4-5 décembre 1998 à l'Université de Paris VII, avec ENS Saint Cloud, Universités de Tours, Limoges et Marne-la-Vallée. Paris : Belin, 2000, p. 81-93.

L'évolution des collections de guides touristiques en langue anglaise au XIX ${ }^{\mathrm{e}}$ siècle, InRevue française de civilisation britannique, vol. XII, $n^{\circ} 4$ (printemps 2004), Mélanges en hommage à Monica Charlot, édités par Antoine Capet, p. 23-35.

Entre continuitéet innovation : les guides touristiques en langue française de 1900 à 1960, In BREUILLOT, Martine et BEAUFILS, Thomas (éds).Le Guide de voyage au fil du Rhin et ailleurs, Actes de la journée d'Études du 19 mars 2004, Université Marc Bloch Strasbourg II ,Départements d'Études Néerlandaises et Néo-helléniques, Université de Strasbourg II, 2005, p. 9-16.

\section{NOTES}

1. - VoirMeyers Reisebücher 1862-1936, Bibliographie von Werner Hauenstein, Ursula Hinrichsen, Stadtoldendorf, 1993.

2. -LISTER, W.B.C.A Bibliography of Murray's Handbooks for Travellers and Biographies of Authors,Editors, Revisers and Principal Contributors. Dereham : Dereham Books, 1993.

3. - MORLIER, Hélène.Les Guides-Joanne. Genèsedes Guides-Bleus. Paris : Les Sentiers débattus, 2007, p. 42. Un index bio-bibliographique sommaire, mais précieux, de 87 auteurs, figure p. 599-607.

4. - Voir MORLIER, Hélène.Les Guides-Joanne. Genèse des Guides-Bleus. Paris : Les Sentiers débattus, 2007, p. 55 et suivantes.

5. - MORLIER, Hélène. Les Guides-Joanne. Genèse des Guides-Bleus. Paris: Les Sentiers débattus, 2007, p. 79. 


\section{RÉSUMÉS}

Après avoir expliqué comment se constitue une collection de guides en relation avec une recherche universitaire, cette contribution décrit le contexte d'apparition des trois grandes collections européennes de guides du XIXe siècle: Murray, Baedeker et Joanne. À travers une analyse comparative très fine, Goulven Guilcher montre ce qui fait leur spécificité et ce qui les distingue. Il s'intéresse aussi à la place que laissent ces collections à d'autres types de guides, à l'influence qu'exercent les auteurs de guides et directeurs de collections sur le contenu de ces publications. L'œil attentif du spécialiste décèle la manière dont les guides de voyage rendent compte des pratiques et des usages touristiques à travers les conseils fournis et les publicités. Le bibliophile, quant à lui, apprécie les cartes et les gravures dont se dotent les guides pour donner à voir les territoires décrits.

After explaining how a collection of guides is built up in connection with research at university level this article studies the genesis of the main three european collections of guidebooks in the XIXth century: Murray, Baedeker and Joanne-Hachette. By means of a detailed comparative analysis GoulvenGuilcher describes the features that characterize them. He also deals with the room left by these collections for other types of guides and the influence exerted by the authors of the guides and the heads of collections over the contents of these publications. By close scrutiny the specialist is able to determine in what way guidebooks take into account the uses of tourism, at the same time by means of advice to travellers and through advertisements. The bibliophile assesses maps and illustrations offeredin the guides in order to give an image of the countries they describe.

\section{INDEX}

Mots-clés : Murray, Baedeker, Joanne, Europe, guides de voyage, guide Reichard, guide Galignani, guide Chaix, guide Garnier, guide Coghlan, guide Leigh, guide Bradshaw, guide Black, guide Meyer, guide Grieben, Thomas Cook, iconographie des guides, cartes, plans, gravures, publicités dans les guides, univers du voyage, collection de guides

\section{AUTEUR}

\section{GOULVEN GUILCHER}

Ancien Professeur Université de Paris XI. Spécialiste des guides et collectionneur, Goulven.guilcher@wanadoo.fr 